\title{
Near field of a vortex generated by chevron-tipped flat plates
}

\author{
Anushka Goyal * and Jovan Nedić ${ }^{\dagger}$ \\ McGill University, Montréal, Québec, H3A OC3
}

Flat plates with chevron tips of variable depths (2h) were designed and their effect on the tip vortex was studied at downstream distances, $x=0.5 \mathrm{c}$ to $3 \mathrm{c}$ at a Reynolds number of 67,000 . The test plates were mounted at an angle of attack of $5^{\circ}$ and the tip vortex was measured using a constant temperature anemometer. The chevron plates formed tip vortices with lower peak tangential velocities and larger core radii as compared to the flat plate (with the exception of the $2 \mathrm{~h}=10 \mathrm{~mm}$, which had a smaller core radius than the flat plate). It was also found that the tip vortices formed over plates with deeper chevrons exhibited turbulent cores, as opposed to the flat plate and shallow chevron plates. Vortex wandering, an inherent meandering of the tip vortex, was found to be present for every test plate. It results in an over-prediction of the core radius and under-prediction of the peak tangential velocities. To ensure that the lower peak tangential velocities and larger core radii of the tip vortices formed over chevron tip plates was due to the change in geometry and not a consequence of vortex wandering, Devenport $e t$ al's correction was applied to the shallow chevron plates and it was found that after correcting for wandering, the tip vortices formed over chevron tips still had lower peak tangential velocities and larger core radii (with the exception of the $2 \mathrm{~h}=10 \mathrm{~mm}$ plate) as compared to the flat plate.

\section{Nomenclature}

$$
\begin{array}{ll}
U_{\infty} & =\text { free stream velocity } \\
\mathrm{c} & =\text { chord } \\
2 \mathrm{~h} & =\text { chevron depth } \\
U_{\theta} & =\text { tangential velocity } \\
r_{c} & =\text { core radius } \\
U_{\theta_{\max }} & =\text { peak tangential velocity } \\
U & =\text { axial velocity }
\end{array}
$$

\footnotetext{
*Graduate Student, Department of Mechanical Engineering, McGill University, 817 Sherbrooke Ouest, Montréal, Québec, H3A 0C3

${ }^{\dagger}$ Assistant Professor, Department of Mechanical Engineering, McGill University, 817 Sherbrooke Ouest, Montréal, Québec, H3A 0C3, AIAA Member.
} 


\section{Introduction}

IP vortices are formed on finite lifting surfaces and are encountered in a wide variety of fluid-body interactions.
Of particular interest are those formed over aircraft wings, as they lead to lift induced drag, thereby reducing the overall aerodynamic performance, increasing carbon dioxide emissions and noise. As well as contributing to the overall drag of the aircraft, tip vortices are coherent structures that persist several chord lengths downstream of the wing, which results in the 'following plane' problem, whereby a trailing aircraft caught in the wake of the leading aircraft is susceptible to reduced control capabilities [1]. It is because of the tip vortex coherence that the minimum distance between aircrafts during take-off and landing at airports is mandated by the Federal Aviation Administration. Methods and designs that decrease the coherence and increase the rate of tip vortex dissipation are therefore desirable in the aviation industry.

The life cycle of tip-vortices can be described by formation (or roll-up), development and the ultimate demise by Crow instabilities. The formation of tip vortices has been attributed by Green [2] to three complimentary theories: a) the pressure difference between the pressure and suction side of a lifting surface leads to a circulatory flow around the wing tips, b) tip vortices form a bridge between the starting vortex and the bound vortex and c) the presence of a shear layer on the surface of the wing. In reality, the formation and development of tip vortices involve complex processes and have been the subject of several studies over the years. After undergoing roll-up, tip vortices show little growth or decay over the first 20 wing-spans downstream of the wing, and the tip vortex shed from one wing develops independently from its counterpart shed by the opposite wing [3]. Finally, it was found by Crow [1], that these tip-vortex pairs will merge and form a single counter-rotating vortex pair, which develops sinusoidal instabilities (or Crow instabilities) and forms a vortex ring that ultimately dissipates. Chevalier [4] found that an aircraft which is in close proximity of a wake that has already formed a vortex ring would face a very small risk of destabilization, as the vortex ring was found to dissipate within 10-20 seconds of its formation.

There has been a concentrated effort in developing methods that would increase the dissipation of tip vortices to address the issue of spacing between aircraft at airports. Jacob et al. [5], for example, used jets in the wing tips to introduce instabilities in the tip vortex in order to facilitate their dissipation; the activation of the jets resulted in an increased span-wise separation between the vortex pairs and an increase in the growth rate of the vortex core. Lee et al. [6] proposed blowing air in the span-wise direction from the wing tip through slots, which deflected the flow around the wing tip outwards, increasing the effective span and displacing the tip vortex outwards. Heyes and Smith [7] combined the two effects by investigating the efficacy of blowing air through slots in the wing tips periodically. By subjecting tip vortices to periodic perturbations at different mass flow rates of air, it was found that the vortex core was larger, peak tangential velocities were lower and axial velocity deficit was larger, as compared to a wing without pulsed jets of air. These changes were a function of the mass flow rate of air through the jets and the the frequency of pulsation, where a high mass flow rate resulted in a weaker tip vortex. Another method that has been found to be 
effective in increasing vortex dissipation rate is increasing the free stream turbulence. Sarpkaya and Daly [8] found that a pair of tip vortices decay at a faster rate when surrounded by a high turbulence intensity due to vortex bursting, as compared to when the vortex pair is surrounded by weak turbulence, where Crow instabilities cause the demise of the vortex pair. Ahmadi-Bloutaki et al. [9] also found that increasing the free stream turbulence from $0.5 \%$ to $4.6 \%$ lead to the formation of a tip vortex on a NACA 0015 wing section with a larger core radius and smaller peak tangential velocity. A similar increase in core radius and decrease in peak tangential velocities in grid generated turbulence were observed by Bailey et al. [10, 11].

The structure of the tip vortex has been shown to be a function of the wing shape and geometry of the wing-tip [12-15]. Incorporating complex geometries into aerodynamic design is a growing area of interest. Multi-scale (fractal-like) geometries, for example, were found to reduce the energy of vortex shedding as compared to their non-fractal counterparts in three-dimensional bluff bodies [16]. Furthermore, chevrons cut into the trailing edge of a NACA 0012 wing were effective in reducing the self noise of the wing [17]. This study was complimented by Nedić et al., [18] who used multi-scale geometries on the trailing edge of a NACA 0012 and found a higher aerodynamic efficiency and lower vortex shedding energy. Prigent et al. [19] studied the effect of multi-scale sinusoidal serrations cut into the trailing edge of a NACA 0012 wing and found a lower energy associated with vortex shedding as well as lower coherence in the spanwise direction. The effect of straight, blunt and serrated trailing edge shapes on a wing were studied through Direct Numerical Simulation and it was found that blunt trailing edges lead to periodic vortex shedding, while serrated trailing edges created a span-wise pressure gradient, leading to a smaller velocity deficit downstream of a trough, as compared to the velocity deficit downstream of a protrusion [20].

The shape of bird wings, specifically, the role of serrated tips of the bird wings (also called 'primaries') has been the subject of several biological studies. Tucker [21] studied the effect of wing-tip slots in a gliding Harris' Hawk and found that the presence of the slots increases the Oswald efficiency number $(e)$ by either influencing the lift distribution, making the wing non-planar or both. More recent work contradicts these findings; vertically separated primary feathers were not found to affect the aerodynamic efficiency in a Gliding Jackdaw during gliding [22]. However, it has been shown that the presence of slotted tips in the Jackdaw lead to the formation of multi-cored tip vortices that resulted in a spread of vorticity [23]. Further, it has been hypothesized that slotted feathers in bird wings evolved to reduce the power cost of take-off and landing in terrestrial or coastal/freshwater birds [24]. Despite contradictory findings on the exact role of slotted wing tips, it remains well established that they lend advantages such as lower drag, enhanced lift, postponement of stall and longitudinal stability to bird flight [21, 25, 26].

Based on these studies, we postulate that slotted feathers act as individual tip vortex generators, where each tip vortex is considerably smaller than the size of the tip vortex that would be created were the feathers not present. Given that the pressure difference between the upper and lower surface changes along the chord, one would expect the strength of each individual tip vortex to be lower; indeed the total combined strength of all the tip vortices could also potentially 
be lower compared to a tip vortex generated across the entire chord of the wing. It is, however, reasonable to assume that at some downstream distance they would merge into a single tip vortex structure. Furthermore, by generating several smaller tip vortices, one can expect a degree of mutual interaction between each vortex, which could alter the dynamics of the tip vortex and potentially reduce its coherence, resulting in faster decay rates. This is similar to the onset of the sinusoidal Crow instabilities in the trailing wake which lead to their eventual breakdown [1].

To test this, we investigate the tip vortex of a flat plate with various chevron designs cut into its tips. Chevrons are a simplistic geometry that can be easily characterized, thus allowing us to test how various parameters of the chevron influence the overall performance. The use of a flat plate, as opposed to an airfoil, allows us to eliminate the added complexity of variable chevron thickness due to the airfoil profile.

\section{Experimental Setup}

\section{A. Chevron Geometry Design}

Tests were conducted on an acrylic flat plate with a span of $300 \mathrm{~mm}$ and a chord of $100 \mathrm{~mm}$, giving a semi aspect ratio $b^{2} / S$ of three, which is similar to the semi aspect ratio observed on birds wings. The thickness of the test plates was $6 \mathrm{~mm}$. A key design requirement was that the planform area, $S$, remain constant in order to facilitate a meaningful comparison between the coefficients of lift and drag. The schematic of the flat plates can be seen in Figure 1 . In order to maintain the same planform area, an integer number of chevrons must be used, where an equal number of triangles are added above and below the mean span line, as shown in Figure 11. Although this method does increase the effective span of the wing by $h$, the mean span of the planform, which we define as $b=S / c$, would remain $300 \mathrm{~mm}$ since the planform area $S$ and the chord length $c$ are unchanged.

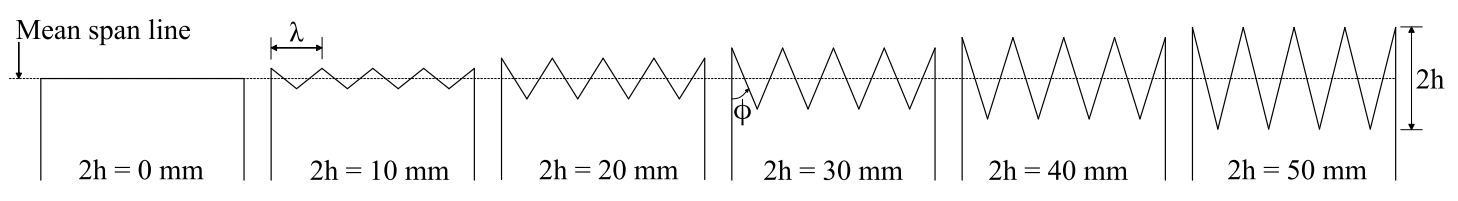

Fig. 1 Chevrons of varying depths $(2 \mathrm{~h}=10,20,30,40$ and $50 \mathrm{~mm})$ cut into a flat plate

A chevron can be characterized by its wavelength $(\lambda)$, depth (2h) and chevron angle $(\phi)$, as shown in Fig 1 . The relationship between these three parameters is given by $\lambda=\tan \phi 4 h$. In this study, the number of chevrons was set to four, whilst the depth $(2 \mathrm{~h})$ was varied and the wavelength was kept constant. Table 1 presents the different plates that were used for this study. 
Table 1 Chevron geometry for each test plate

\begin{tabular}{lccccc}
\hline Plate Number & Wavelength $(\lambda)(\mathrm{mm})$ & $2 h(\mathrm{~mm})$ & Number of Chevrons & Chevron Angle $\phi\left(^{\circ}\right)$ & Symbol \\
\hline 1 & $\infty$ & 0 & 0 & $90.00^{\circ}$ & $\star$ \\
2 & 25 & 10 & 4 & $32.00^{\circ}$ & $\star$ \\
3 & 25 & 20 & 4 & $22.60^{\circ}$ & $\square$ \\
4 & 25 & 30 & 4 & $17.30^{\circ}$ & $\bullet$ \\
5 & 25 & 40 & 4 & $14.08^{\circ}$ & $\checkmark$ \\
6 & 25 & 50 & 4 & $11.76^{\circ}$ & $*$ \\
\hline
\end{tabular}

\section{B. Wind Tunnel Setup and Methodology}

All measurements were taken in the Newman Wind Tunnel in the Aerodynamics Lab of the Department of Mechanical Engineering, McGill University. The Newman Wind Tunnel is an open circuit tunnel with a rectangular, closed test section of cross section $2 \mathrm{ft} \times 3 \mathrm{ft}$ and a working length of $6 \mathrm{ft}$. The longitudinal background turbulence intensity is $1 \%$ at a freestream velocity of $U_{\infty}=10 \mathrm{~m} / \mathrm{s}$. The chord based Reynolds number for all the tests was $\operatorname{Re}_{c}=U_{\infty} c / v=67,000$. The blockage ratio with the plate orientated perpendicular to the flow ( $\alpha=90^{\circ}$ in Figure 2 ) is 5.3\%, with the value decreasing for lower angles of attack; as such, no blockage corrections were applied [27]. Literature has shown that while the presence of free-stream turbulence does not affect the initial formation of a tip vortex or the position of the vortex center, it does affect the tangential velocity profiles [9, 11]; it should be noted that these findings were observed for significantly larger freestream turbulence values than the one found here. Furthermore, the effects of background turbulence can be neglected as the objective of this study is to draw a comparison between the different test plates, all of which are subjected to the same background turbulence. The plate was set to an angle of attack of five degrees.

The velocity field of the tip vortex was measured using an Auspex Four Sensor Vorticity probe (diameter of 5 microns and a sensor length of $1 \mathrm{~mm}$ ), connected to an AA Labs AN-1003 Constant Temperature Anemometer. A sketch of the setup and the co-ordinate system is shown in Figure 2. To locate the center of the tip vortex, we followed Ahmadi-Baloutaki et al. [9] and traversed the hot-wire over a 33mm x 33mm grid in the $y-z$ plane, from $z$ values ranging from $-0.1<z / c<0.23$ and $y$ values ranging from $-0.26<y / c<0.07$, with $1 \mathrm{~mm}$ increments in both directions. The resolution of the traverse was $0.02 \mathrm{~mm}$. The trailing edge of the test plates at an angle of attack of $0^{\circ}$ was chosen as the the global origin $(0,0,0)$, as shown in Figure 2 Grid measurements were taken at downstream distances of $x=0.5 \mathrm{c}$, $1 \mathrm{c}, 1.5 \mathrm{c}, 2 \mathrm{c}, 2.5 \mathrm{c}$ and $3 \mathrm{c}$ from the global origin. The center of the tip-vortex was identified as the point where the time averaged $V$ and $W$ components of velocity are zero [9]. Having identified the vortex center, the hot-wire probe was traversed in the $y$ direction in $1 \mathrm{~mm}$ increments to measure the tangential velocity profiles. Data were sampled at $30 \mathrm{kHz}$ for 60 seconds, and low-pass filtered at $14 \mathrm{kHz}$. The probe uncertainty was calculated using the method proposed by Bendeict and Gould [28], and was found to be $0.8 \%$ for $U_{x}, 2.5 \%$ for $U_{\theta}, 1 \%$ for $u_{r m s}, 1.6 \%$ for $v_{r m s}$ and $1.9 \%$ for $w_{r m s}$. Corrections for tip vortex wandering shall be addressed in SectionVII 


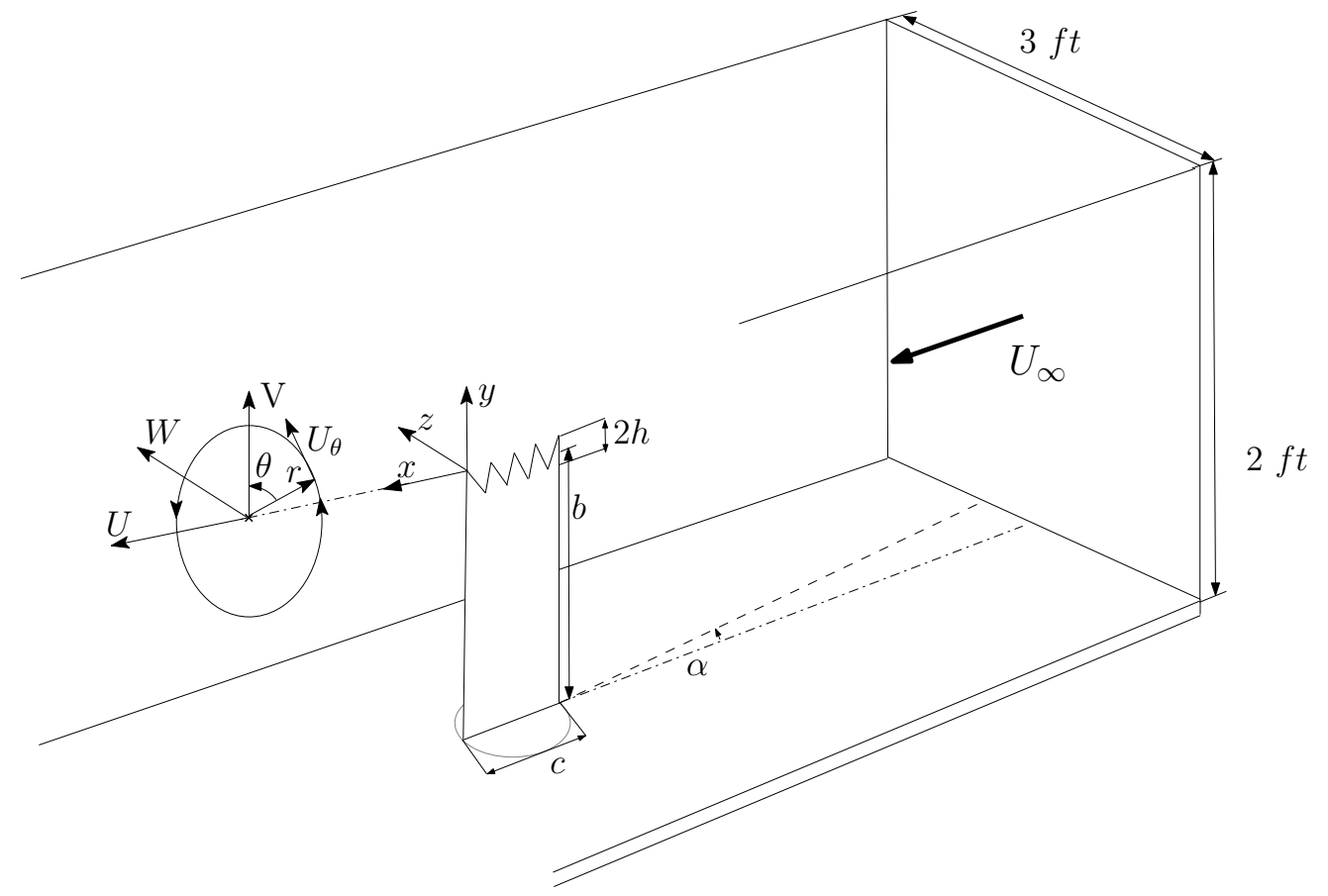

Fig. 2 Sketch of the setup in the Newman Wind Tunnel, where $x$ is the streamwise component and $y$ and $z$ are the planar components

\section{Position of the Tip Vortex}

Figure 3 shows the position of the vortex center in the $y$ and $z$ directions at the five downstream positions considered here. As the chevron depth increases, one can observe that the tip vortex center is shifted inboard (Figure 3a) and lifted upwards (Figure 3b. In previous studies [9, 11], the inboard and upward shift in the position of the vortex center with streamwise distance in the formation stage was attributed to the roll-up of the shear layer [29], whilst the presence of free-stream turbulence did not affect the position of the tip vortex.

\section{Mean Tangential Velocity Profiles}

The radial profile of the tangential velocity, $U_{\theta}$, at $\mathrm{x}=0.5 \mathrm{c}$ and $1 \mathrm{c}$ for all the test plates is shown in Figure 4 . As we move radially away from the vortex centroid, the tangential velocity first increases, reaching a peak tangential velocity at a radial location known as the core radius, $r_{c}$, before decreasing again. The region from the vortex centroid to $r_{c}$ is known as the vortex core. In this study, the radial profile of the tangential velocity at $x=0.5 \mathrm{c}$ for all the test plates exhibited a double core structure, indicated by a dual peak in the profile shown in Figure 4a Devenport et al. [30] similarly observed a double-core structure in the near field of a NACA0012 wing and argued, based on the observations of Engel [31], that these double peaks arise due to the presence a strong, co-rotating secondary tip vortex from the pressure side of the wing-tip, that ultimately merges with the primary vortex formed over the suction side. Figure $4 \mathrm{~b}$ shows the radial profile of the tangential velocity at $x=1 \mathrm{c}$, where only a single peak can be observed in the profiles, 


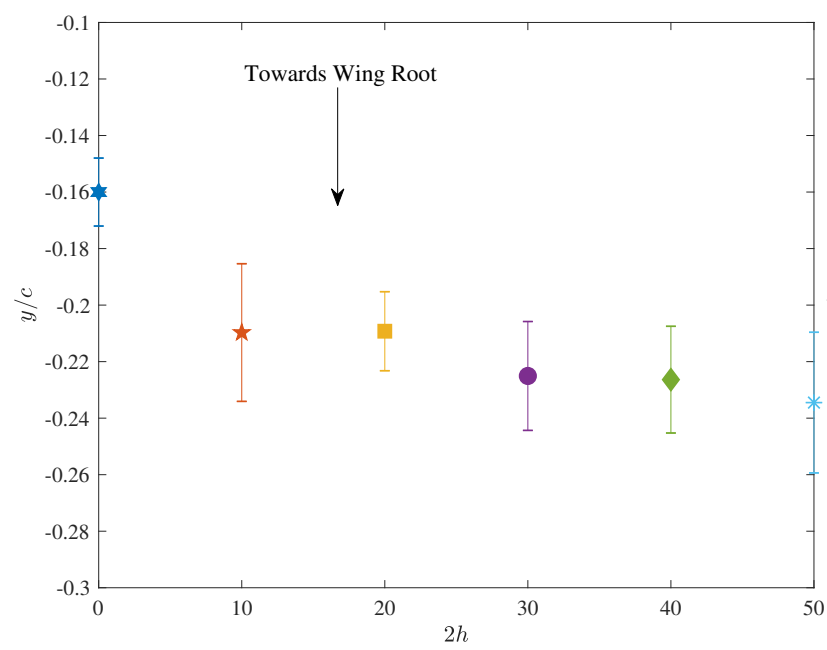

(a)

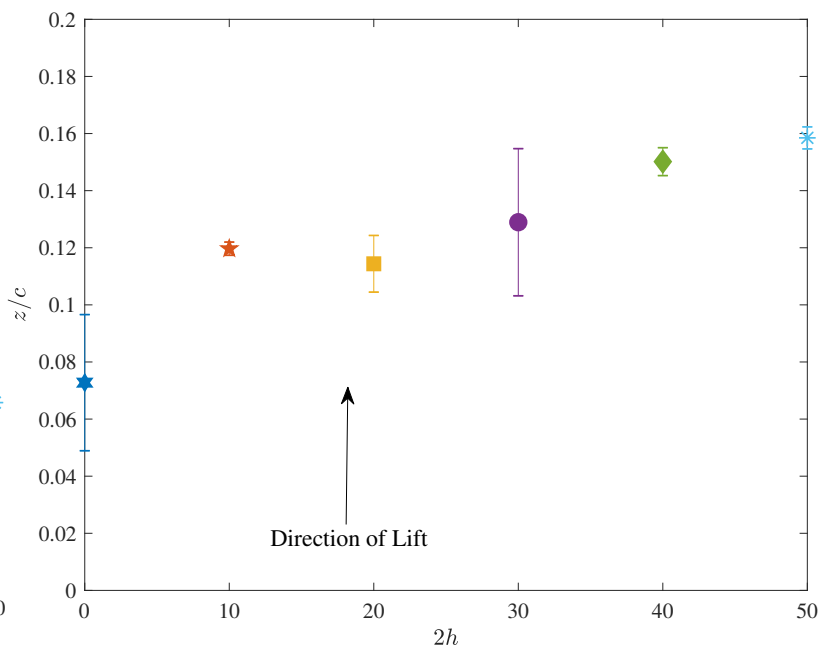

(b)

Fig. 3 Vortex center position in the $y$ and $z$ direction (see Figure 2)

which is again in-line with the observations of Devenport et al. [30] and one can assert that primary and secondary vortices must have merged by $\mathrm{x}=1 \mathrm{c}$. The presence of multiple vortices during the initial development of a tip vortex and their subsequent merging has been documented by several studies. Development of a tip vortex formed on a rounded NACA 0012 wing-tip by Chow et al. [32] has shown the merging of tertiary and secondary vortices with the primary vortex within one chord length from the trailing edge. Furthermore, Bailey et al. [11] highlighted that squared wing tips form a tip vortex due to three distinct vortices merging together: one along the wing-tip, the second on the suction side and the third at the trailing edge of the wing.

The self-similar nature of the vortex core can be observed by normalizing the tangential velocity with the peak tangential velocity, $U_{\theta_{\max }}$, and the radial distance by the core radius. Self similarity of the vortex core is given by Equation 1 [33] and is shown in Figure 5 for each test plate for $x=1 \mathrm{c}$ to $3 \mathrm{c}$. It is evident that the tip vortex shows self similarity up to $r_{c}<1.2$, consistent with the literature [9, 11, 33], which has highlighted that this region of the tip vortex is self similar regardless of the levels of free-stream turbulence and vortex wandering (corrected and uncorrected). Therefore, the peak tangential velocity and core radii can be used as indicators of how the tip geometry affects the vortex core. One can also observe from Figure 5 that the normalized tangential velocity profiles fan out at $r / r_{c}>1.2$, similar to what is observed when a tip vortex is subjected to grid turbulence [9, 34]. This aspect shall be investigated further in a later section.

$$
\frac{U_{\theta}}{U_{\theta_{\max }}}=\frac{1}{0.716\left(\frac{r}{r_{c}}\right)}\left[1-\exp \left(-1.2526\left(\frac{r}{r_{c}}\right)^{2}\right)\right]
$$

Figure 6a shows the peak tangential velocities for each test plate, normalised by the free stream velocity at all five downstream positions. The peak tangential velocities for the chevron plates are significantly lower than the flat plate, 


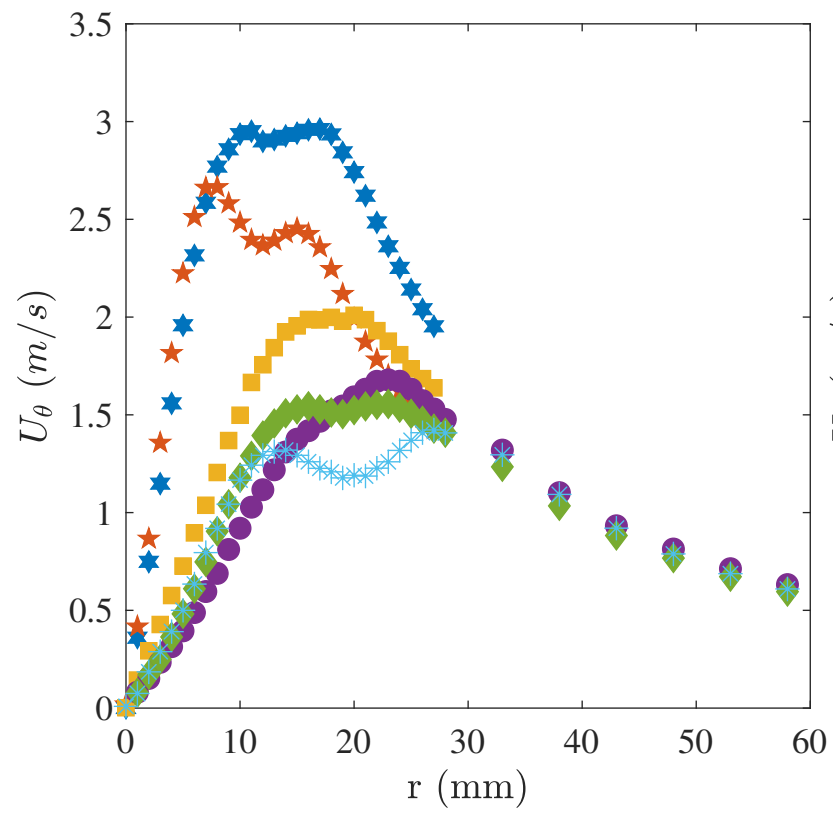

(a) $x / c=\mathbf{0 . 5}$

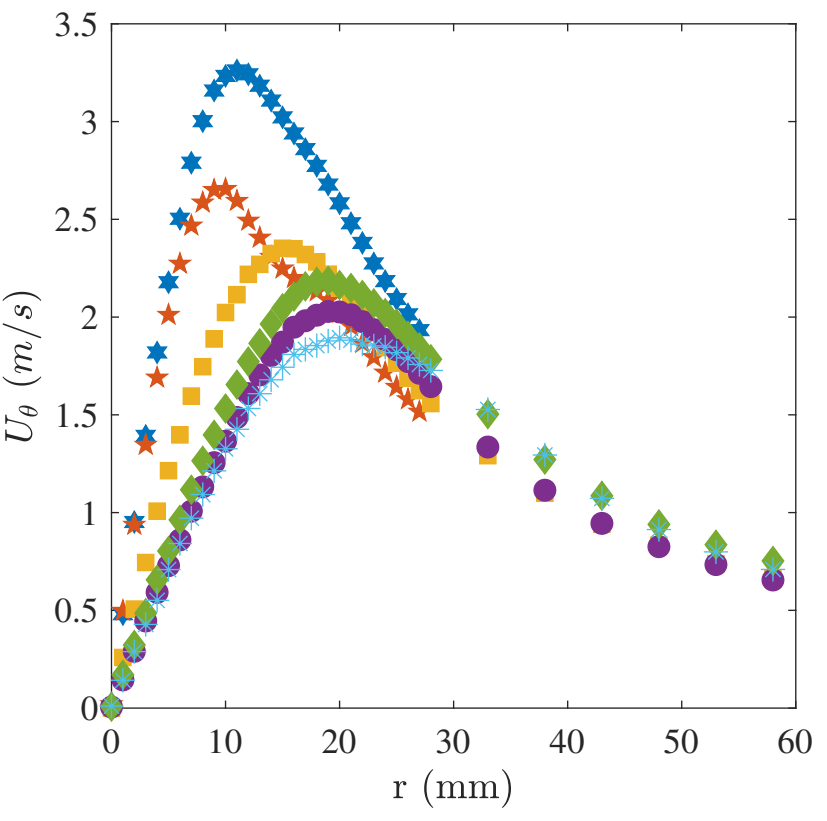

(b) $x / c=1$

Fig. 4 Tangential Velocity profiles at a) $0.5 \mathrm{c}$ and b) $1 \mathrm{c}$, showing a double core structure at $0.5 \mathrm{c}$, which merges into a single core at $x<1 c$

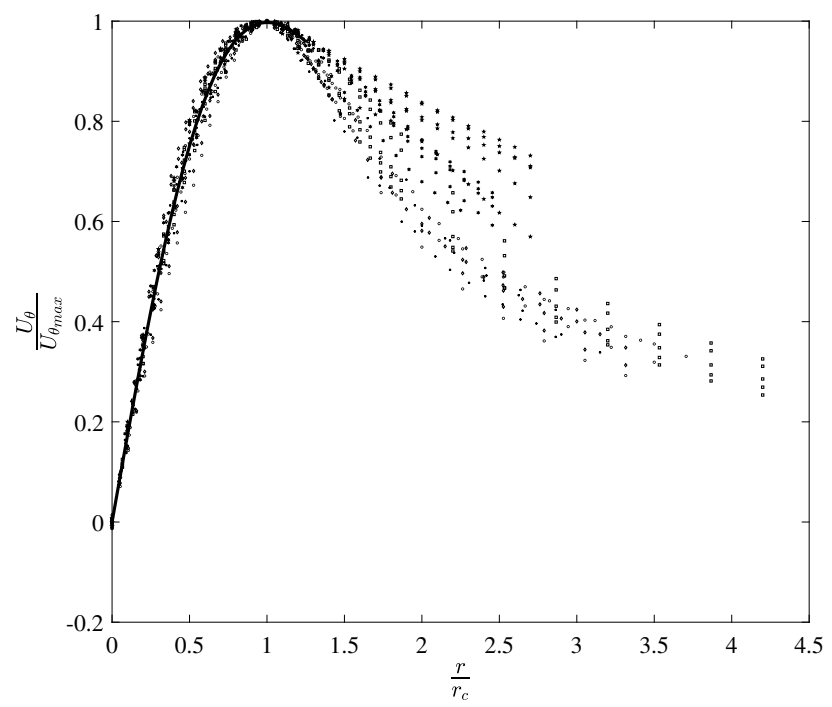

Fig. 5 Normalised tangential velocity, $U_{\theta} U_{\theta \text { max }}$, as a function of normalised radial distance, $r r_{c}$, for all plates and downstream locations. Solid line indicates analytical solution by Phillips [33] 


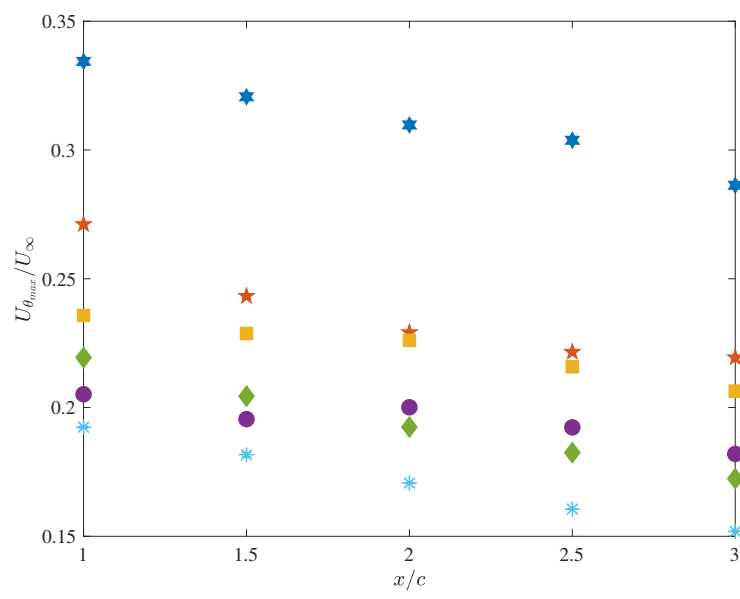

(a)

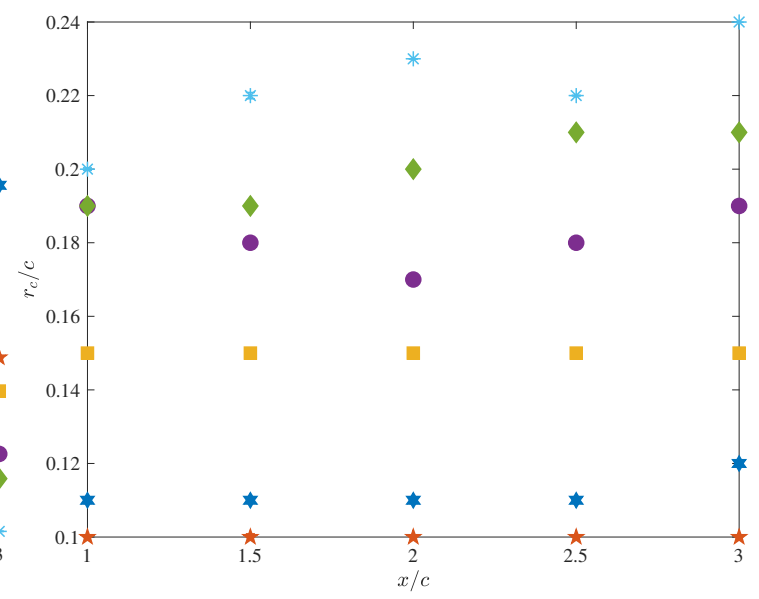

(b)

Fig. 6 Streamwise variation of (a) peak tangential velocity $U_{\theta \max }$ normalised by freestream velocity $U_{\infty}$, and (b) vortex core radius, $r_{c}$, normalised with the chord. See Table 1 for symbols

implying a weaker core, with the peak velocity decreasing as $2 \mathrm{~h}$ (chevron depth) increases. For example, the $2 \mathrm{~h}=10$ $\mathrm{mm}$ chevron wing produced roughly a $20 \%$ reduction in peak tangential velocity compared to the flat plate, whilst the $2 \mathrm{~h}=40 \mathrm{~mm}$ had a $35 \%$ reduction compared to the flat plate.

The core radius $\left(r_{c}\right)$ is another key parameter in characterizing the tip vortex. Figure 6b shows the core radii of each test plate, normalized by the chord length. The core radius for the chevron plates is larger than that of the flat plate, with the exception of the $10 \mathrm{~mm}$ chevron plate, which exhibits a $10 \%$ reduction in core radii. The $30 \mathrm{~mm}$ chevron plate, for instance, shows a $36 \%$ increase in the core radius at $x=3 \mathrm{c}$, as compared to the flat plate. It can also be observed, in the case of deeper chevron plates $(2 \mathrm{~h}=30 \mathrm{~mm}, 40 \mathrm{~mm}$ and $50 \mathrm{~mm})$, that the core radii increase for a given plate with an increase in downstream distance. Studies have shown that the core radius is a function of the geometry of the wing tip. For instance, Sarpkaya [13] observed that the radius of a tip vortex generated by a rectangular planform with a square cut tip is 30\% larger than that of the same planform with a rounded tip. Shekarriz et al. [14] found that this increase in the radius of the tip vortex is due to the formation of the primary vortex from several shear layer vortices that are generated at the tip, which merge downstream. Stinebring et al. [12] found that the axial velocity in the core decreases and core size increases on roughening a smooth, round wing-tip. Giuini [35] showed that a squared wing tip produces a higher number of smaller vortices as compared to rounded tips.

One can conclude that chevron tip test plates exhibit a decrease in peak tangential velocities and increase in core radius as compared to the flat plate. A given chevron plate also shows a larger core radius with an increase in streamwise distance. These characteristics, in addition to the fanning out of normalised tangential velocity profiles at $r>1.2 r_{c}$ are similar to that of a tip vortex subjected to free-stream turbulence [9-11].

The combined effect of a lower peak tangential velocity and larger core radius (except for the $2 \mathrm{~h}=10 \mathrm{~mm}$ plate) is evaluated by integrating the non-dimensional radial velocity profile, defined as $\xi=\int_{0}^{3} U_{\theta} / U_{\infty} d\left(r / r_{c}\right)$, which we use as 


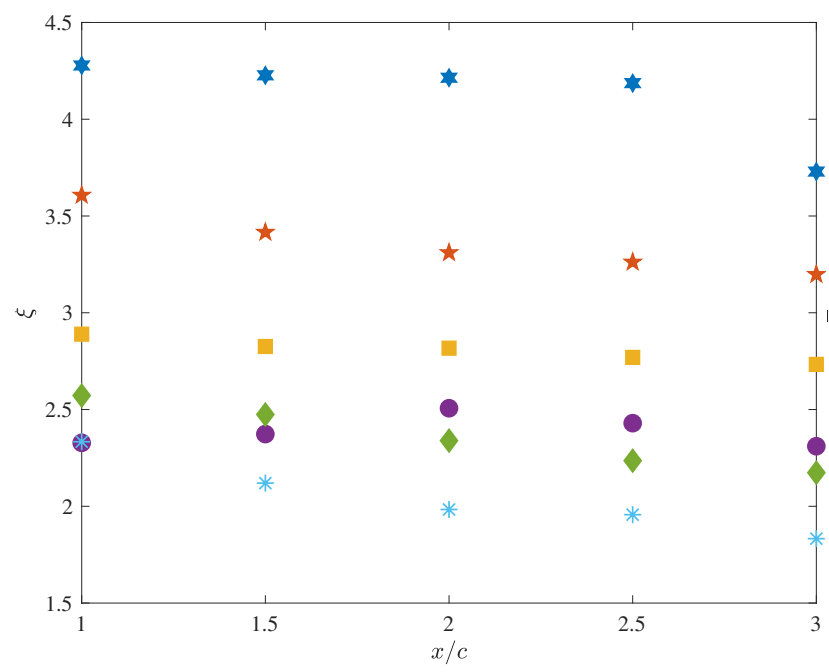

(a)

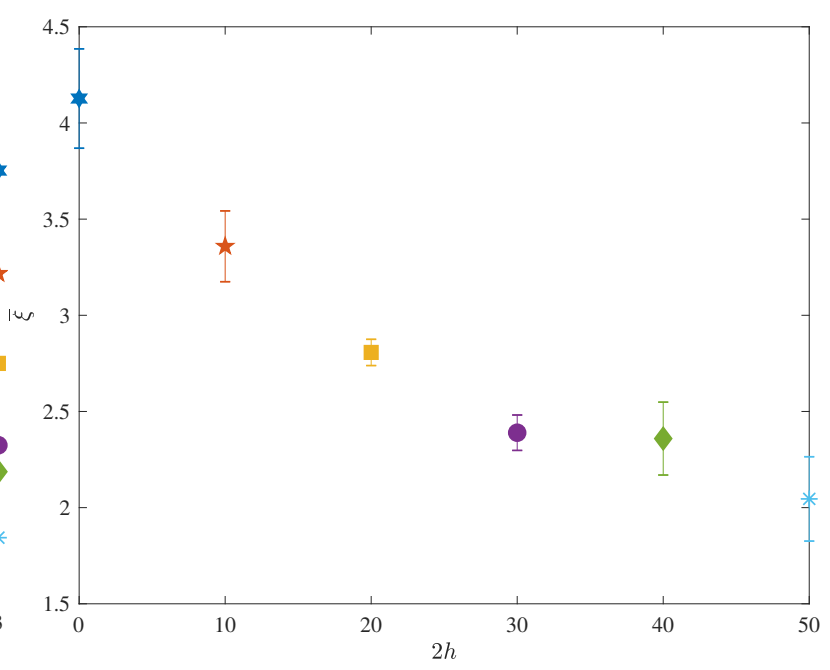

(b)

Fig. 7 Streamwise variation of tip vortex strength $\xi$ for all plates showed in (a). Average vortex strength as a function of chevron depth shown in (b). See Table 1 for symbols.

a measure of vortex strength. If the area under the $U_{\theta}-r$ curve was equal for the flat plate and the chevron plates, it would indicate that the chevrons altered the radial distribution of the tangential velocity without altering the overall vortex strength. The data are shown in Figure7a. Three times the core radial distance was chosen because it includes a sufficient portion of the outer core region, where the tangential velocity profiles seem to roughly collapse (shown in Figure $4 \mathrm{~b}$. We note from Figure $7 \mathrm{a}$ that the values of $\xi$ are roughly constant over the downstream range considered here, and hence in Figure $7 \mathrm{~b}$ we show the average $\xi, \bar{\xi}$, as a function of chevron depth. From Figure $7 \mathrm{~b}$, a lower value of $\bar{\xi}$ is observed for an increase in chevron depth. For instance, the $\bar{\xi}$ value for the $2 \mathrm{~h}=50 \mathrm{~mm}$ plate is $63 \%$ lower than the flat plate. The lower $\bar{\xi}$ values, therefore, indicate a weaker tip vortex for the chevron plates. Furthermore, it should be noted that even though the core radius of the $2 \mathrm{~h}=10 \mathrm{~mm}$ plate is smaller than that of the flat plate, when the overall

effect of this reduction in core radius and peak tangential velocity is considered using $\bar{\xi}$, the $2 \mathrm{~h}=10 \mathrm{~mm}$ plate lies between the flat plate and the $2 \mathrm{~h}=20 \mathrm{~mm}$ plate.

\section{Frequency Spectra at the Vortex Center}

Having demonstrated that the chevrons decrease the strength of the tip vortex, we now turn our attention to investigating possible reasons for this decrease in strength. We begin by investigating the frequency spectrum at the core of the vortex to determine if the flow is laminar or turbulent. The frequency spectrum of the streamwise velocity component, $F_{u u}$, at the time averaged vortex centre is shown in Figure 8 The streamwise velocity component was chosen as the $v$ and $w$ components could potentially show a turbulent core due to vortex wandering, which is a low frequency transverse motion of the vortex in time, which we shall expand on in Section VII

From Figure 8 , one can see that the slope of the spectra for $2 \mathrm{~h}=0 \mathrm{~mm}$ plate is closer to a value of -3 for $\mathrm{x}>0.5 \mathrm{c}$, 
which is indicative of a laminar vortex core. A slope of -3 was also observed by Devenport et al. [30] for a laminar vortex core. At $x=0.5 c$, the slope of the energy spectra, over a very narrow range of frequencies, is close to $-5 / 3$, therefore, indicating that the vortex core is turbulent in the very near field. Hence we can conclude that the vortex core for the flat plate is initially turbulent (albeit weakly), and eventually laminarizes further downstream.

For the $2 h=10 \mathrm{~mm}$ chevron plate (Figure $8 \mathrm{p}$ ), the slope of the spectra tends to-5/3 at $\mathrm{x}=0.5 \mathrm{c}, 1.0 \mathrm{c}$ and $1.5 \mathrm{c}$, again over a very narrow range of frequencies, and tends to -3 as the downstream distance increases. The change in slope of the frequency spectra from $-5 / 3$ to -3 as one moves downstream indicates that the vortex core is turbulent-like for a larger downstream distance from the wing tip as compared to the flat plate. A discernable peak is observed for the $2 \mathrm{~h}=$ $10 \mathrm{~mm}$ chevron plate at $440 \mathrm{~Hz}$, which decreases with downstream distance. This peak is assumed to be associated with vortex shedding, and has a Strouhal number, based on the thickness of the plate $(\mathrm{t}=6 \mathrm{~mm})$ and freestream velocity, of $S t=f t / U_{\infty}=0.26$. The $2 \mathrm{~h}=20 \mathrm{~mm}, 30 \mathrm{~mm}, 40 \mathrm{~mm}$ and $50 \mathrm{~mm}$ chevron plates exhibit a turbulent core at every downstream position considered here, suggesting that deeper chevrons create a turbulent vortex core. The fact that the vortex cores for these plates are turbulent would explain why the tip vortex is both wider and weaker, since turbulence results in increased levels of mixing and diffusion, as compared to a laminar core.

Having identified that the core is turbulent, we now turn our attention as to why this is the case. From the literature, a turbulent core has been attributed to two possible phenomena: the tip vortex is still in its initial formation stage [36] or vortex wandering [30]. Since the farthest measurement plane is $3 \mathrm{c}$ downstream of the trailing edge, it is possible that the tip vortices are undergoing roll-up. It has been shown by Katz et al. [36] that a turbulent core during the initial formation is due to the wing boundary layer as well as large axial velocities in the vortex core. However, there is no unanimous agreement on the criteria used to determine when the vortex roll-up process is complete. Using the criteria of an invariant tangential velocity and circulation with downstream distance, Green and Acosta [37] found that the roll-up process was complete- for a rectangular wing with a square tip- within 2-3 chordlengths downstream of the trailing edge. Shekkariz et al. [38] used an uncambered rectangular wing and found that the roll-up process was complete at $x / c$ $<1$. Contrary to these studies, Phillips [33] asserted that the vortex roll-up process is complete once the spiral wake is no longer distinct from the vortex core; therefore, it continues for several chord lengths downstream of the wing. Therefore, it cannot be said with certainty whether or not the tip vortices measured in this study are undergoing roll-up. Nevertheless, from our data we can assume that the $2 \mathrm{~h}=0 \mathrm{~mm}$ and $10 \mathrm{~mm}$ plates do undergo the initial formation phase since their spectra are at $-5 / 3$ and then tend towards -3 as we move downstream. However, the same can not be said for the other plates. It appears, however, that the $-5 / 3$ frequency range is increasing with downstream distance, and so the core is not laminarizing, as was the case the previous two plates. Therefore, for the $2 \mathrm{~h}=20,30,40$ and $50 \mathrm{~mm}$ plates, the vortex core is turbulent at all downstream locations. This would therefore imply that the formation process for these tip vortices must have occurred between $x=0.5 c$, where a double core is evident (see Figure 4a) and 1c, where a single core is observed (see Figure 4b). 
A possible explanation for the persistent turbulent core for the higher chevron depth plates is vortex wandering, which shall be investigated in the following sections.

\section{Cross Power Spectral Density Coefficient}

Tip vortices are susceptible to a random, low frequency motion called wandering, which has been attributed to free stream turbulence [11, 30, 39], instabilities within the vortex core such as the ejection of fluid packets from the core [2], vibration of the vortex generator [40] and boundary conditions [41]. Wandering leads to an over-prediction of the core radius and under-prediction of the peak tangential velocities in measurements made by fixed probes, such as hot wire anemometry [7, 10]. The amplitude of wandering has also shown to increase with an increase in downstream distance [30]. Devenport et al. [30] proposed an iterative analytical solution to correct the tangential and axial velocity profiles for wandering under the assumption that the true position of the vortex center can be described by a non-isotropic Gausian probability density function. This assumption was validated by Heyes et al. [7].

Vortex wandering is a planar phenomenon that affects both the $v$ and $w$ components of velocities simultaneously. Therefore, to study the effects of chevrons on vortex wandering, the cross power spectral density coefficient $\left(F_{v w}^{2} / F_{v v} F_{w w}\right)$, using the $v$ and $w$ velocity components at the vortex center, was computed and is shown in Figure 9 To facilitate a comparison between the raw, measured frequencies for this study and other studies, a 'reduced frequency' was defined using $f_{r}=f c / U_{\infty}$.

The cross power spectral density coefficient at the vortex center for the flat plate shows peaks over a wide range of measured frequencies, the most discernable ones being at $10 \mathrm{~Hz}, 26 \mathrm{~Hz}, 120 \mathrm{~Hz}$ and $300 \mathrm{~Hz}$ (corresponding to a reduced frequency, $f_{r}$ of $0.1,0.26,1.2$ and 3 , respectively). There is no agreed threshold frequency for vortex wandering, however, for this study, it is assumed that wandering taken place at frequencies lower than $100 \mathrm{~Hz}$. A threshold of 100 Hz was also used by Bailey et al. [10], who argue that the wandering motion is associated with frequencies 'significantly lower' than frequencies associated with turbulent activity. From Figure 8 , we note that the emergence of the $-5 / 3$ inertial range, a typical indicator for turbulence, occurs at frequencies higher than $100 \mathrm{~Hz}$. This corresponds to a reduced frequency, $f_{r}$ of 1 , and we note that the reduced frequency for vortex wandering for Bailey et al. [10] was similarly equal to 1 . Note that the $2 \mathrm{~h}=10 \mathrm{~mm}$ chevron plate shows a sharp peak at $440 \mathrm{~Hz}$, which diminishes with an increase in streamwise distance; this is shown in Figure 8 and is presumed to be associated with vortex shedding. For the $2 \mathrm{~h}=10$ $\mathrm{mm}$ plate at $x=2 \mathrm{c}$, we begin to observe a peak over a broad band of frequencies, roughly centred about $10 \mathrm{~Hz}\left(f_{r}=0.1\right)$, that increases with streamwise distance. The emergence of this low frequency, attributed to vortex wandering, coincides with the streamwise location where the spectra for this plate show a laminarization of the vortex core (see Figure $8 \mathrm{p}$ ). In general, the coefficient of cross power spectral density for the $10 \mathrm{~mm}$ plate shows peaks over a range of frequencies that are wider than the $20 \mathrm{~mm}$ plate, but narrower than the flat plate. For the deeper chevron plates $(2 \mathrm{~h}=20,30,40$ and $50 \mathrm{~mm}$ ), we see distinct peaks over a narrow range of frequencies, centered about a raw frequency of $50 \mathrm{~Hz}$, or a 

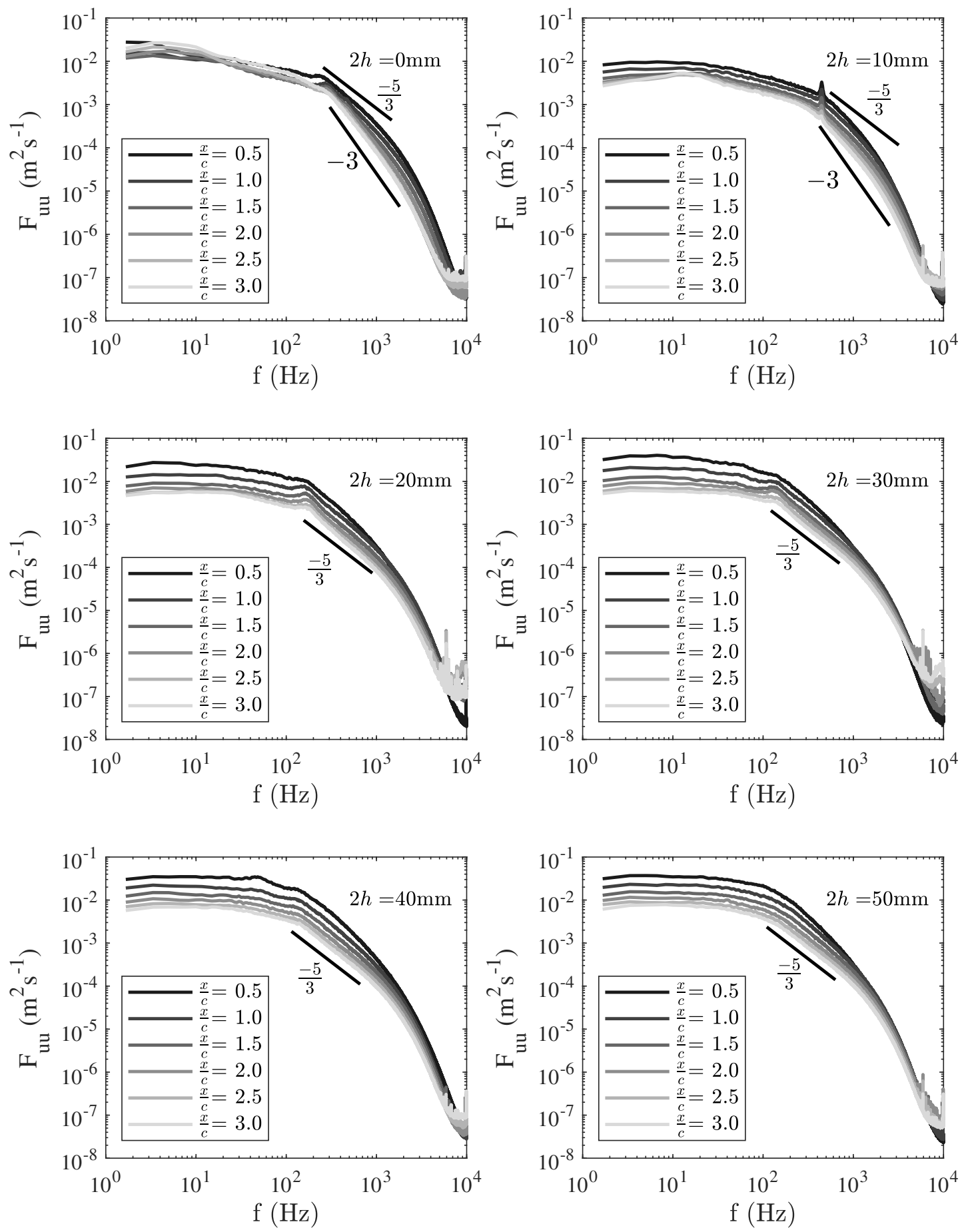

Fig. 8 Frequency Spectra for $2 \mathrm{~h}=0 \mathrm{~mm}, 2 \mathrm{~h}=10 \mathrm{~mm}, 2 \mathrm{~h}=20 \mathrm{~mm}, 2 \mathrm{~h}=30 \mathrm{~mm}, 2 \mathrm{~h}=40 \mathrm{~mm}$ and $2 \mathrm{~h}=50 \mathrm{~mm}$ chevron plates at various downstream positions. 

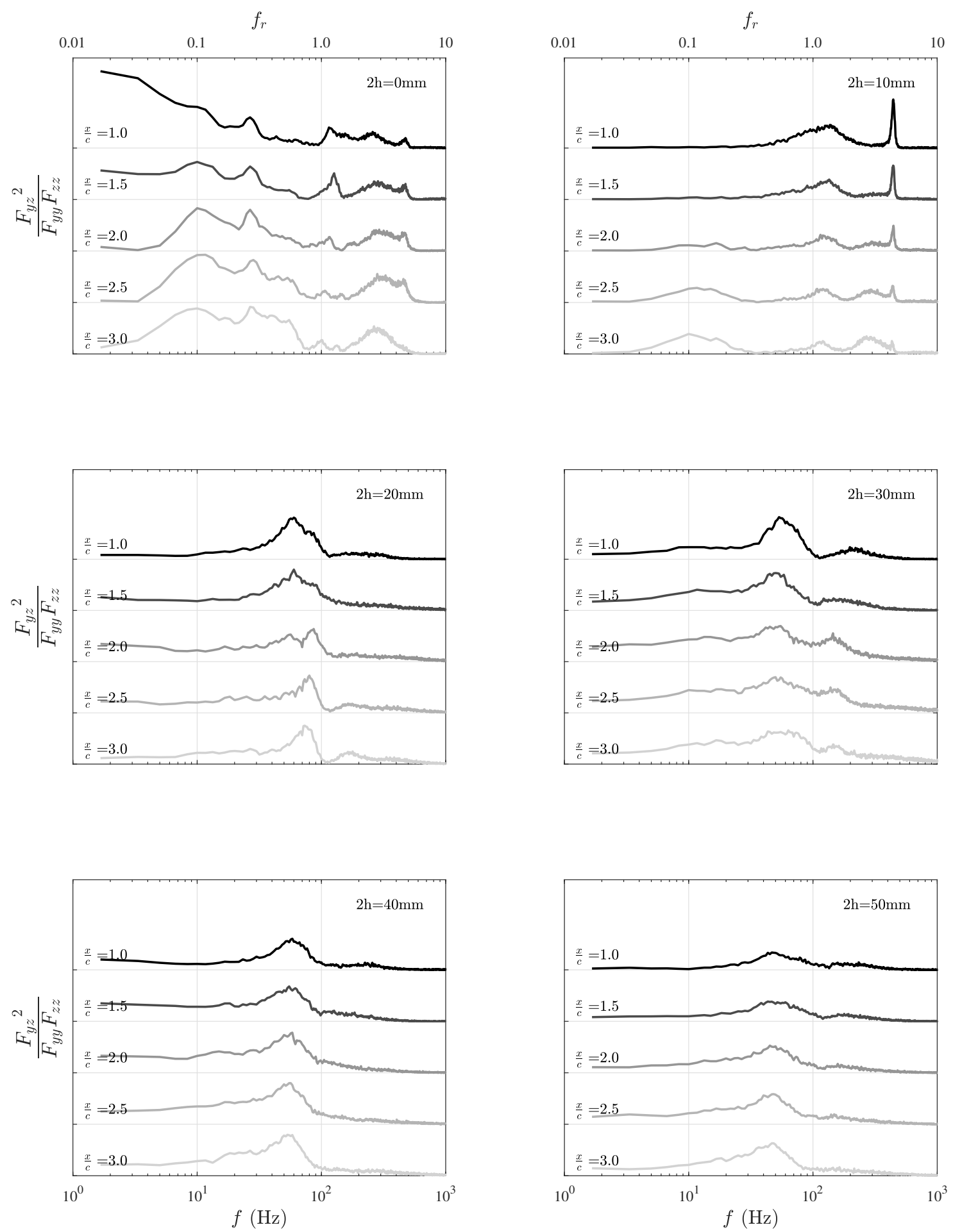

Fig. 9 Cross Power Spectral Density Coefficient for all the test plates at various downstream positions on a semi-log scale 
reduced frequency of 0.5 . This reduced frequency is close to the value of 0.42 obtained by Bailey et al. [10] for vortex wandering in small and large free-stream grid generated turbulence for a downstream distance of $x=3.75 \mathrm{c}$. Finally, for the deeper chevron plates, the peak associated with what is presumed to be vortex shedding is mostly absent (this is also corroborated by the $u$ velocity spectra shown in Figure 8). One can speculate that the presence of deeper chevrons suppresses vortex shedding, however, this requires further investigation through flow visualization.

From the coefficient of cross power spectral density, we can infer that the contribution of energy at the vortex center is from vortex wandering at frequencies lower than $100 \mathrm{~Hz}$ (or $f_{r}$ less than 1) and turbulence in the core at frequencies larger than $100 \mathrm{~Hz}$. From the perspective of a comparison between the vortex wandering for chevron plates and the flat plate, it can be inferred that the motion of the vortex center for the deeper chevron plates has a dominant frequency, as opposed to the flat plate, which shows multiple peaks at frequencies lower than $100 \mathrm{~Hz}$. While the exact effect of the chevrons on the turbulent activity in the tip vortex and the wandering of the vortex requires further investigation, it is evident that the cross spectra for the chevron plates is very different from that of the flat plate. Therefore, by extension, one can speculate that the wandering associated with deeper chevron plates could potentially be more organised as compared to the $2 \mathrm{~h}=0$ and $10 \mathrm{~mm}$ plates. The cross spectra also indicates that vortex shedding is absent in the deeper chevron plates.

\section{Devenport's Correction}

Having identified that the tip vortex experiences vortex wandering, we now address means by which its effects can be corrected. Devenport et al.'s [30] method is an iterative technique that requires the measured velocity profiles taken along the $z$ axis to be fitted by a series of the form:

$$
U_{\theta}\left(0, z_{p}\right)=\sum_{i=1}^{n} \frac{D_{i}}{z_{p}}\left[1-\exp \left(\frac{-z_{p}^{2}}{c_{i}}\right)\right]
$$

This method assumes that the position of a wandering vortex center can be described by a non-isotropic Gaussian probability density function, where the standard deviations are the wandering amplitudes, $\sigma_{y}$ and $\sigma_{z}$ and $e$ is the correlation coefficient. The coefficient $D_{i}$ in Equation 2 is obtained using a least square curve fit, while $c_{i}$ is guessed for the first iteration. To avoid complex results, the value of $c_{i}$ was set above $\left[2 \sigma_{z}^{2}\left(1-e^{2}\right)\right]^{\frac{1}{2}}$. The corrected tangential velocity profiles are then obtained and a comparison is made between the measured and corrected Reynold's stresses at the vortex center. If the difference is more than the tolerance (set at $0.1 \%$ ), then the process is repeated with new values for $\sigma_{y}$ and $\sigma_{z}$. The corrected peak tangential velocities and core radii are shown in Figures 10a and 10b, respectively.

It is observed that the corrected peak tangential velocities are higher than the measured ones. Similarly, the core radii are smaller, when corrected for wandering. However, as compared to the flat plate, the chevron plates have lower peak tangential velocities and larger core radii, even after the wandering correction is applied (with the exception of the 


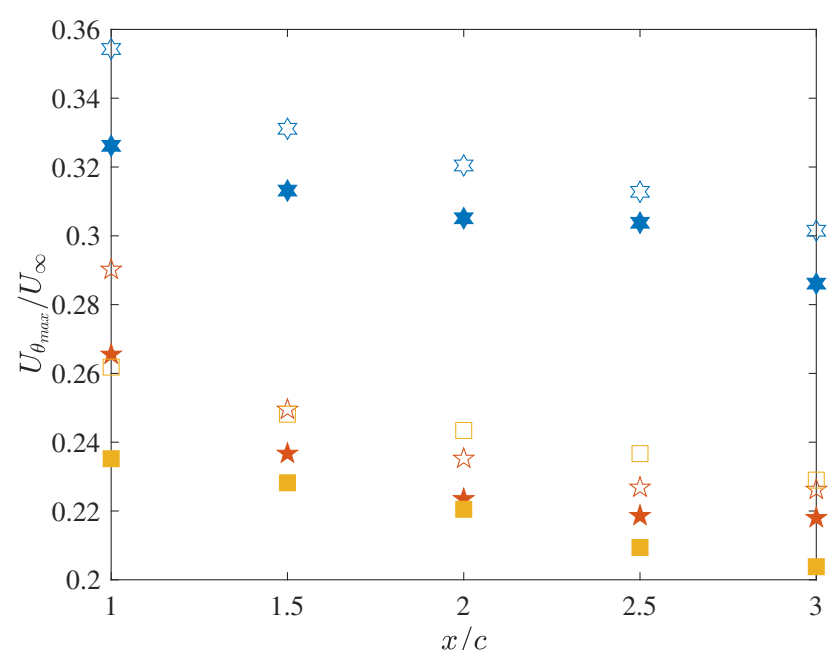

(a)

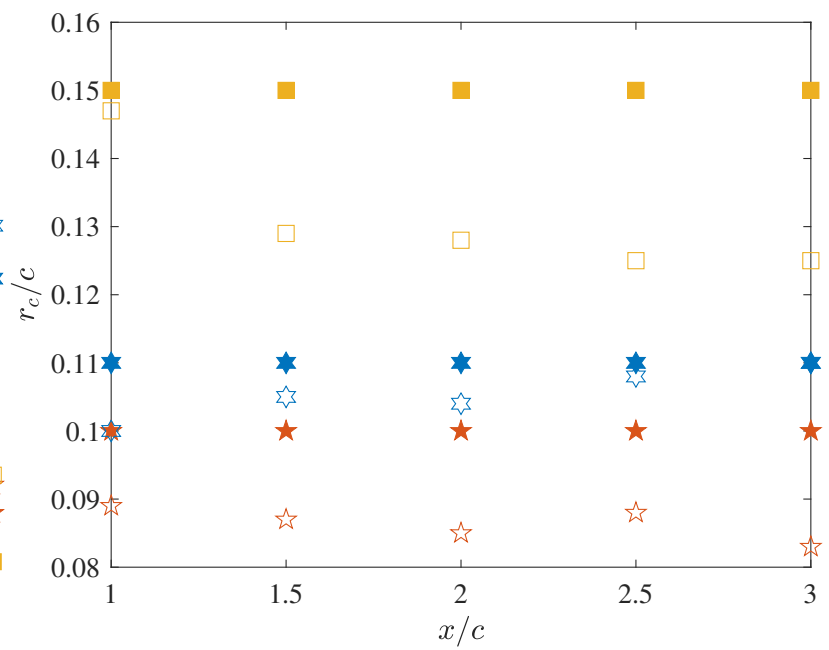

(b)

Fig. 10 a) Measured (solid symbols) and Corrected (hollow symbols) peak tangential velocities normalized by the free stream velocity and b) Measured (solid symbols) and Corrected (hollow symbols) core radii normalized by the chord length

$2 \mathrm{~h}=10 \mathrm{~mm}$ plate, where the corrected core radius is smaller than that of the flat plate). Therefore, despite accounting for wandering, qualitatively, the presence of chevrons leads to smaller peak tangential velocities and larger core radii as compared to the flat plate. Following an analysis similar to what was presented in Section IV $\xi$ and $\bar{\xi}$ were computed for the corrected velocity profiles and are shown in Figure 11 . Since the corrected peak tangential velocities are larger than the measured ones, it can be expected that $\xi$ values would follow the same trend, i.e., the values of $\xi$ for the corrected profiles are larger than the ones for the measured profiles. Therefore, the spatial average of $\xi$, given by $\bar{\xi}$ is larger for each test plate than the measured counterparts. For instance, corrected $\bar{\xi}$, for the $2 \mathrm{~h}=20 \mathrm{~mm}$ plate is $10.8 \%$ larger than the measured $\bar{\xi}$.

It has been shown that Devenport et al.'s method was accurate when $\sigma_{y} / r_{c}$ and $\sigma_{z} / r_{c}$ were less than 60\% [42]. On applying this correction to the $2 \mathrm{~h}=0,10$ and $20 \mathrm{~mm}$ plates, the wandering amplitudes normalised by the core radius were found to increase with chevron depth as well as downstream distance, while remaining within $40 \%$. The increase in $\sigma_{y} / r_{c}$ and $\sigma_{z} / r_{c}$ with increase in chevron depths and downstream distances is significant because vortex wandering in atmospheric turbulence aids in faster dissipation of tip vortices [9, 10]. Since atmospheric turbulence intensity cannot be changed at will, the only direct means by which the wandering of the vortex can be altered is by changing the geometry of the lifting surface i.e., the addition of chevrons, which contributes to vortex dissipation.

This correction could not be applied to the velocity profiles for the deeper chevron plates i.e., for $2 \mathrm{~h}=30,40$ and 50 $\mathrm{mm}$, as Devenport et al.'s correction is valid only for a laminar core, and the deeper chevron plates exhibited a turbulent core. On applying the correction to the deeper chevron plates, convergence was not achieved for a tolerance of $0.1 \%$. When the tolerance was raised to $1 \%$, convergence was achieved, however, the solution was sensitive to the number of 


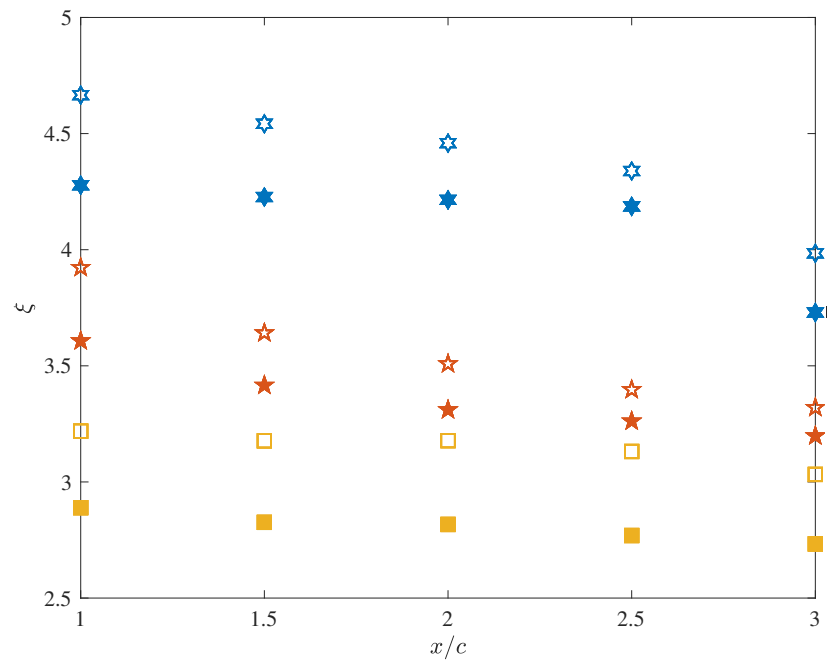

(a)

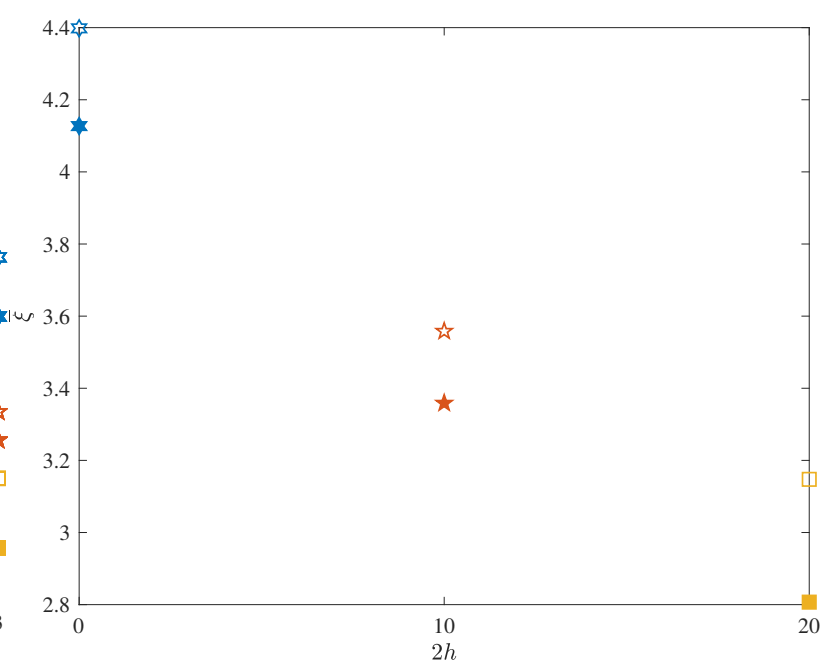

(b)

Fig. 11 Measured values (solid symbols) and corrected values (hollow symbols) of a) $\xi$ and b) $\bar{\xi}$

terms, $n$, in the series fit. Smaller values of $n$ lead to poor fits. At larger wandering amplitudes, the minimum value of $c_{i}$ exceeded the core radius. It was also observed that a small amount of scatter in the measured data lead to a large oscillation in the corrected profile. These results were also observed by Bailey et al. [10] when they attempted to correct the tangential velocity profiles for wandering for a high free-stream turbulence case. Despite the fact that a wandering correction could not be applied to the deeper chevron plates, the trend observed when the correction is applied to the shallower chevron test plates is promising and indicative of a weaker tip vortex due to the presence of chevrons. For the $\bar{\xi}$ value of the $2 \mathrm{~h}=50 \mathrm{~mm}$ plate to be equal to that of the flat plate, a $100 \%$ correction would be required, which seems unlikely based on the fact that the $2 \mathrm{~h}=10$ and $20 \mathrm{~mm}$ plates required a correction of $5.6 \%$ and $10.8 \%$, respectively.

\section{Mean Axial Velocity and Turbulence Intensity Profiles}

Having identified that deeper chevron plates have a turbulent core, it is of interest to investigate the mean and turbulence profiles, so as to gain a deeper understanding of the tip vortex structure itself. Tip vortices have been shown to exhibit either an axial velocity deficit [30] i.e., 'wake-like' (or have a deficit with respect to the free stream), or an axial velocity surplus [32] i.e., 'jet-like' (higher than the free stream velocity) in the core. Batchelor [43] showed analytically that the nature of the axial velocity is determined by the balance between the radial circulation gradient and the dissipation of momentum: if the latter is greater than the former, the axial velocity is wake-like. Anderson and Lawton [15] found that the axial velocity depends on the angle of attack of the wing and the tip shape: wings with a rounded tip and a high angle of attack lead to a jet like flow, as opposed to wings with a square tip and at low angle of attack, which form a wake like axial velocity profile. Therefore, it was of interest to study the effect of chevron tips on the axial velocity profiles of the tip vortices. 
In Figure 12, the normalized mean axial velocity deficit profiles for each test plate at every downstream position are shown. An axial velocity deficit within the vortex core is observed for all cases, that tends towards the free stream and plateaus thereafter as we move radially away from the vortex center. Furthermore, it is observed that the axial velocity deficit at the vortex center decreases for each test plate with an increase in the streamwise distance. Figure 13 shows the normalised axial velocity at the vortex center for each chevron plate. The centreline axial velocity deficit for the deeper chevron plates decreases more rapidly compared to the shallower chevron plates.

The normalised turbulent kinetic energy is calculated using $K=0.5\left(\overline{u^{\prime 2}}+\overline{v^{\prime 2}}+\overline{w^{\prime 2}}\right) / U_{\infty}^{2}$ and is shown in Figure 14 There is a discernable difference in the $K$ profiles of the test plates. At $x=1 \mathrm{c}$, the normalised turbulent kinetic energy increases up to at $0.5 r_{c}$ and then decreases for each test plate. However, as the downstream distance increases, the $K$ profiles of the deeper chevron plates $(2 \mathrm{~h}=20,30,40$ and $50 \mathrm{~mm})$ deviate significantly from the $2 \mathrm{~h}=0$ and $10 \mathrm{~mm}$ plates. The deeper chevron plates show a constant value of $K$ up to $0.5 r_{c}$ and then tends to zero as the radial distance from the vortex center increases. In contrast, the normalised turbulent kinetic energy of the flat plate and $10 \mathrm{~mm}$ chevron plates decreases with an increase in radial distance from the vortex center and tend to zero more slowly than the deeper chevron plates. The deeper chevron plates, therefore, have a very distinct profile, as opposed to the $0 \mathrm{~mm}$ and $10 \mathrm{~mm}$ chevron plates. This is corroborated by the fact that the $2 \mathrm{~h}=0$ and $10 \mathrm{~mm}$ plates exhibit a non-turbulent-like power spectrum in the core and throughout.

It can be observed for the deeper chevron plates from Figures 13 and 14 that a decrease in the axial velocity deficit (as streamwise distance increases) is accompanied by a decrease in the normalised kinetic energy. For instance, the $2 \mathrm{~h}=$ $50 \mathrm{~mm}$ chevron plate exhibits a turbulent vortex centre at each downstream position and large values of $K$. Its axial velocity deficit is the largest at $x=1 \mathrm{c}$ and the deficit decreases to that of the flat plate at $x=3 \mathrm{c}$. Contrary to this, the flat plate exhibits a laminar core from $x=1 \mathrm{c}$ to $3 \mathrm{c}$ and has the smaller values of $K$ as compared to the chevron plates. Its axial velocity deficit is the lowest at $x=1 \mathrm{c}$ and decreases with streamwise distance at a rate lower than the chevron plates. Clearly, the rate at which the axial velocity deficit decreases for the test plates is linked to the normalised turbulent kinetic energy.

\section{Discussion}

The main objective of this study was to evaluate the impact of chevron wing tips on the strength of the tip vortex in the near field, and it has been shown that increasing the chevron depth resulted in the tip vortex becoming weaker by lowering the peak tangential velocities and increasing the core radius (except the $2 \mathrm{~h}=10 \mathrm{~mm}$ plate, which had a smaller core radius). This was further illustrated by using a non-dimensional integral of the velocity profile, $\xi$, which gave significantly lower values for the $2 \mathrm{~h}=20,30,40$ and $50 \mathrm{~mm}$ plates as compared to the flat plate and $2 \mathrm{~h}=10 \mathrm{~mm}$ plate (see Figure 7a. As shown in Figure 8, the deeper chevron plates had a slope of $-5 / 3$ at every downstream position and the frequency range increased with downstream distance, indicating that the core was not laminarizing, which was the 

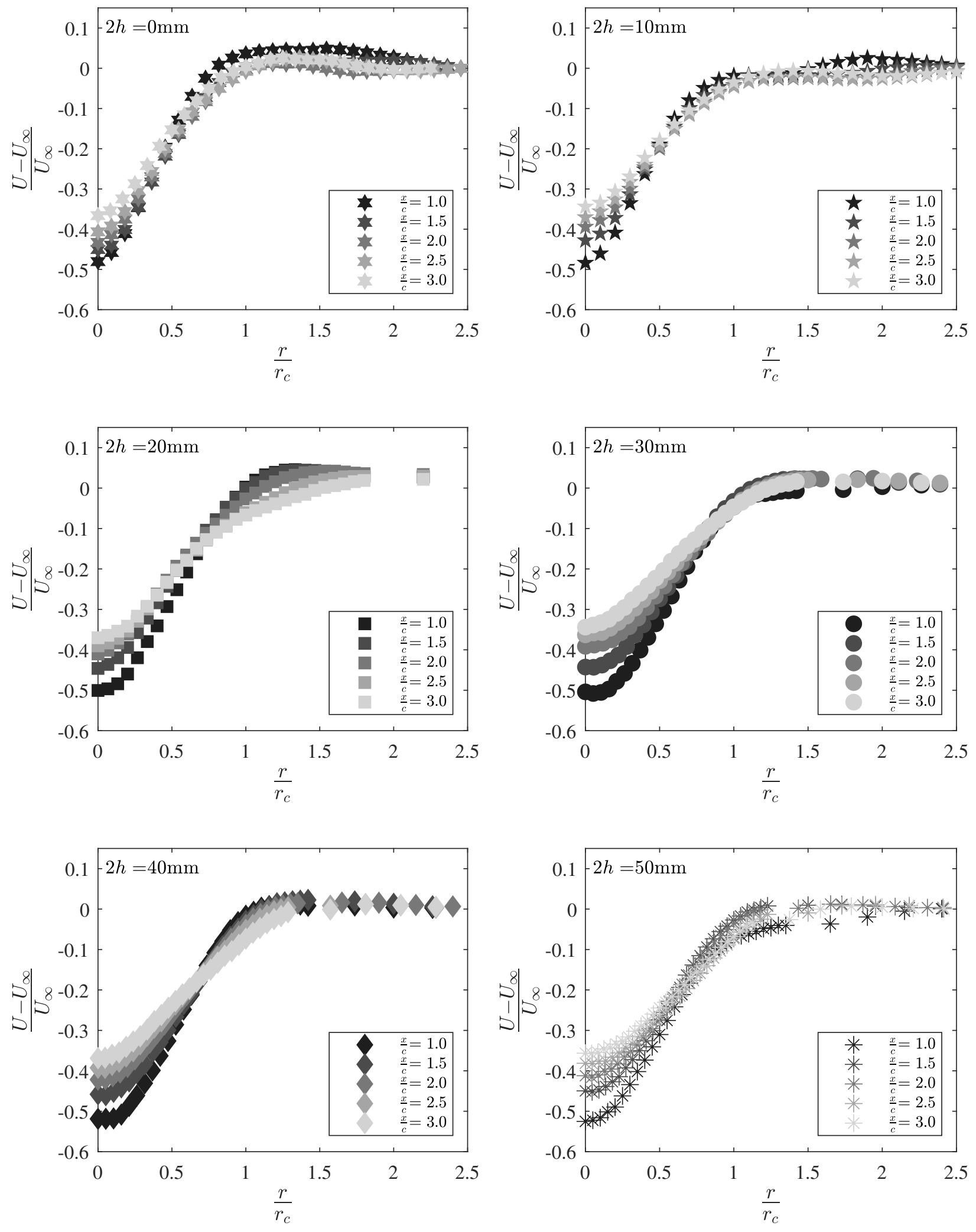

Fig. 12 Normalized Axial Velocity Deficit $\left(U_{x}-U_{\infty}\right) / U_{\infty}$ for each test plate at $x / c=1.0,1.5,2.0,2.5$ and 3.0 


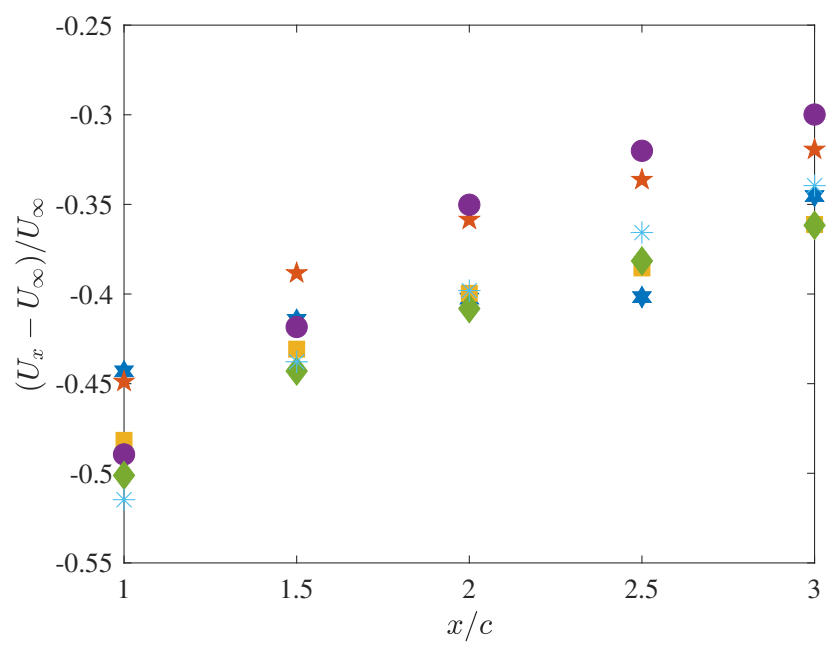

Fig. 13 Normalized Axial Velocity Deficit $\left(U_{x}-U_{\infty}\right) / U_{\infty}$ for each test plate at $x / c=1.0,1.5,2.0,2.5$ and 3.0.

case for the shallower chevron plates, but was fully turbulent. Vortex wandering and initial formation effects were both shown not to be the cause of the turbulent core in the deeper chevron plates, whilst the turbulent profiles - see Figure 14 - clearly indicated that the deeper chevron plates exhibit radial distinctive profiles, different to the shallower chevron plates. Combined, the results indicate that the tip vortices for the deeper chevron plates are turbulent. Therefore, the wider and weaker tip vortex could be attributed to the turbulent nature of the core, as it would lead to enhanced levels of mixing and diffusion.

To demonstrate this, we compare the average strength of the tip vortex i.e., $\bar{\xi}$ from Figure $7 \mathrm{~b}$ to the average total core kinetic energy within our measurement range i.e., $\overline{K^{\prime}}$, where $K^{\prime}=\int_{0}^{1} K d\left(r / r_{c}\right)$, which is shown in Figure 15 The figure shows a clear trend: as the chevron depth increases, the turbulent kinetic energy increases within the core and the tip vortex becomes weaker, i.e., the value of $\bar{\xi}$ decreases. Finally, it is of interest to note which component of the turbulent kinetic energy is the largest contributor to $K^{\prime}$ so as to discern which component is the primary driver of the weakening of the tip vortex. In Figure 16, we show $K_{u}^{\prime}, K_{v}^{\prime}$ and $K_{w}^{\prime}$, where $K_{u}^{\prime}=\int_{0}^{1} 0.5 \overline{\left(u^{\prime}\right)^{2}} / U_{\infty}^{2} d\left(r / r_{c}\right)$, with $K_{v}^{\prime}$ and $K_{w}^{\prime}$ calculated in a similar manner. It is evident from Figure 16 that $\overline{u^{\prime 2}}$ has the largest contribution to $K^{\prime}$ ( $58 \%$ at $\mathrm{x}=1 \mathrm{c})$, followed by $\overline{v^{\prime 2}}(24 \%$ at $\mathrm{x}=1 \mathrm{c})$ and $\overline{w^{\prime 2}}(18 \%$ at $\mathrm{x}=1 \mathrm{c})$. As downstream distance increases to $\mathrm{x}=3 \mathrm{c}$, the contribution of $\overline{u^{\prime 2}}$ to $K^{\prime}$ decreases to $45 \%$, while that of $\overline{v^{\prime 2}}$ increases to $37 \%$ and $\overline{w^{\prime 2}}$ remains fairly constant. There is, therefore, a significant decrease in $K_{u}^{\prime}$ with an increase in downstream distance as compared to the $v$ and $w$ components. Furthermore, at $\mathrm{x}=1 \mathrm{c}$, it is observed that the value of $K_{u}^{\prime}$ increases with an increase in chevron depth (with the exception of the $2 \mathrm{~h}=10 \mathrm{~mm}$ plate). For example, there is an $69 \%$ increase in the value of $K_{u}^{\prime}$ between the $2 \mathrm{~h}=0 \mathrm{~mm}$ and $50 \mathrm{~mm}$ chevron plates. One can therefore argue that the primary reason the tip vortex becomes weaker is due to an initial substantial increase in streamwise kinetic energy. Given that this increase in streamwise kinetic energy occurs very close to the flat plate, and that there is a clear increase in $K_{u}^{\prime}$ as chevron depth increases, the source of this increase 

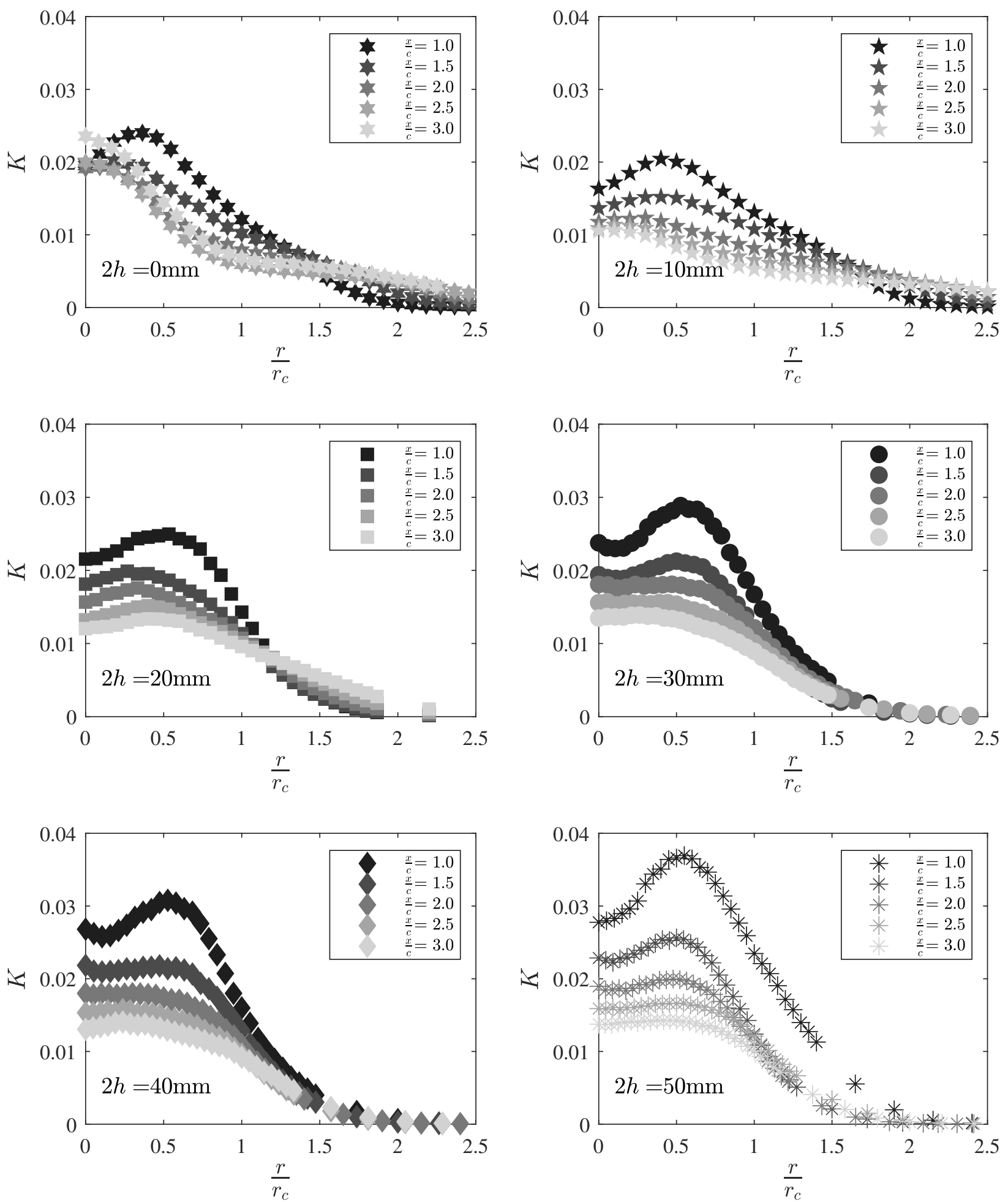

Fig. $14 K$ for each test plate at each downstream position 


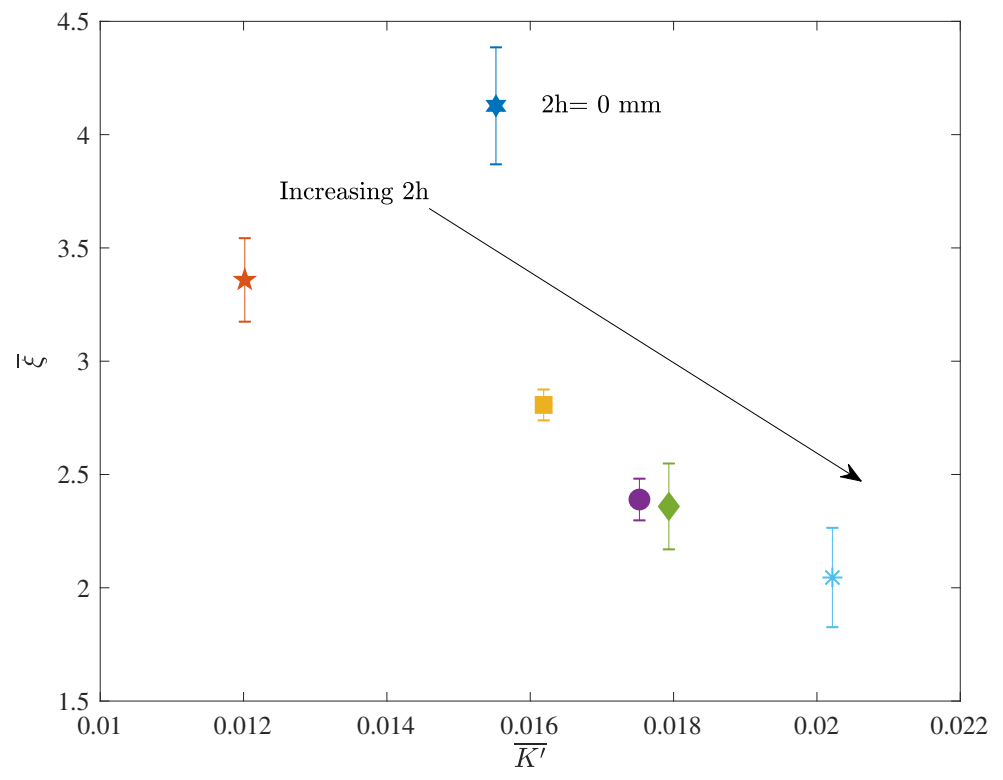

Fig. 15 Normalized vortex strength, $\xi$, as a function of core turbulence intensity, $\bar{K} \prime$.
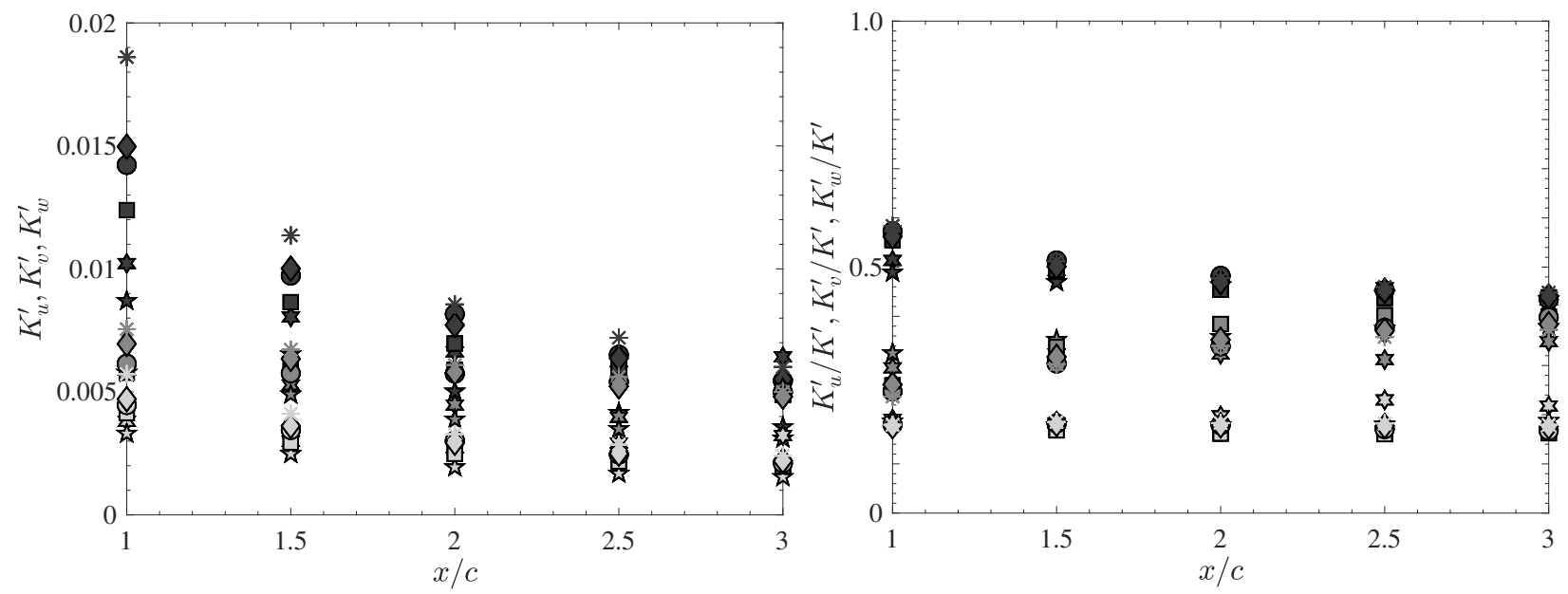

Fig. 16 Streamwise variation of a) $K_{u}^{\prime}$ (solid), $K_{v}^{\prime}$ (gray) and $K_{w}^{\prime}$ (light) and b) $K_{u}^{\prime} / K^{\prime}$ (solid), $K_{v}^{\prime} / K^{\prime}$ (gray) and $K_{w}^{\prime} / K^{\prime}$ (light) for each test plate 
must be the chevrons themselves. This increase due to chevron depth, however, diminishes as downstream distance increases and at $\mathrm{x}=3 \mathrm{c}$, the difference is negligible. The presence of chevrons, however, does not significantly alter the contribution of $K_{u}^{\prime}, K_{v}^{\prime}$ and $K_{w}^{\prime}$ to $K^{\prime}$, i.e., when each component of $K^{\prime}$ is normalised by the total kinetic energy within the core, the curves for all the test plates, except the most upstream location, collapse.

\section{Conclusions}

Vortices formed over chevron tips on a flat plate were found to have lower peak tangential velocities and larger core radii (with the exception of the $2 \mathrm{~h}=10 \mathrm{~mm}$ plate). The overall strength of the vortices was computed using an integral of the normalised tangential velocity over the radial distance up to three times the core and was found to be smaller for deeper chevron plates. The weaker tip vortices were attributed to turbulence in the core. The frequency spectra of the streamwise velocity component was found to exhibit a slope of $-5 / 3$ for deeper chevron plates $(2 \mathrm{~h}=20,30,40$ and 50 $\mathrm{mm}$ ), and were hence turbulent, while that of the $2 \mathrm{~h}=0$ and $10 \mathrm{~mm}$ plates had a slope of -3 and were therefore laminar. The cross power spectral density coefficient showed that the vortex was wandering for each test plate. Correction to wandering was applied to the $2 \mathrm{~h}=0,10$ and $20 \mathrm{~mm}$ plates, but could not be applied to the deeper chevron plates as Devenport et al's [30] correction is not valid for a turbulent core. On applying the correction it was found that vortex wandering did lead to an under-prediction of the tangential velocities and over-prediction of the core radii, however, despite the correction, the chevron plates exhibited a lower peak tangential velocity and a larger core radius than the flat plate (with the exception of the $2 \mathrm{~h}=10 \mathrm{~mm}$ plate). Furthermore, the wandering amplitudes of the chevron plates were larger than those of the flat plate, which could potentially be used to aid in vortex dissipation. The wandering 'signature' of the flat plate and $2 \mathrm{~h}=10 \mathrm{~mm}$ chevron plates were similar as they exhibited peaks over a wide range of frequencies. Contrary to this, the deeper chevron plates exhibited a spike at a single dominant frequency. Mean axial velocity profiles were wake-like for every test plate, with the deeper chevron plates exhibiting a larger velocity deficit at the vortex center. The normalised turbulent kinetic energy profiles of the deeper chevron plates were very distinct and different from the laminar, shallow chevron plates. In addition, the integral of the turbulent kinetic energy was found to be larger for the deeper chevron plates, which is used to explain the lower strength of the tip vortices- a turbulent core would result in increased levels of turbulent mixing and diffusion, leading to a weaker tip vortex. Despite the fact that the $2 \mathrm{~h}=$ $50 \mathrm{~mm}$ plate gives the weakest tip vortex, it should be reiterated that this study focused on chevron geometries with four chevrons and a fixed wavelength. Understanding the exact effect of chevron geometry variation on the tip vortex requires further analysis examining both of these variables, as well as the effect of airfoil cross-section. Furthermore, the stability and far-field development of the tip vortices needs to be studied to understand why some geometries are better than others. 


\section{Acknowledgements}

This study would not have been possible without funding from Fonds de recherche du Québec - Nature et technologies (FRQNT). The authors would like to thank Stavros Tavoularis from the University of Ottawa for lending the AA Labs anemometer used for this study.

\section{References}

[1] Crow, S., "Stability theory for a pair of trailing vortices," AIAA Journal, Vol. 8, No. 12, 1970, pp. 2172-2179. https: //doi.org/10.2514/3.6083

[2] Green, I., Fluid Vortices, Springer Science+Business Media Dordrecht, 1995.

[3] Iversen, J. D., “Correlation of turbulent trailing vortex decay data,” Journal of Aircraft, Vol. 13, No. 5, 1976, pp. 338-342. https://doi.org/10.2514/3.44529. URL https://doi.org/10.2514/3.44529

[4] Chevalier, H., "Flght test studies of the formation and dissipation of trailing vortices," Journal of Aircraft, Vol. 10, 1973, pp. 14-18. https://doi.org/10.2514/3.60193.

[5] Jacob, J. D., Liepmann, D., and Savas, O., "Natural and forced growth characteristics of the vortex wake from a rectangular airfoil," AGARD CP 584, 1996.

[6] Lee, C. S., Tavella, D., Wood, N. J., and Roberts, L., "Flow structure and scaling laws in lateral wing-tip blowing," AIAA Journal, Vol. 27, 1988, pp. 1002-1007. https://doi.org/10.2514/3.10211.

[7] Heyes, A. L., and Smith, D. A. R., "Spatial perturbation of a wing-tip vortex using pulsed span-wise jets," Experiments in Fluids, Vol. 37, 2004, pp. 120-127. https://doi.org/10.1007/s00348-004-0791-5.

[8] Sarpkaya, T., and Daly, J. J., "Effect of ambient turbulence on trailing vortices,” Journal of Aircraft, Vol. 24, 1987, pp. 399-404. https://doi.org/10.2514/3.45459

[9] Ahmadi-Baloutaki, M., Carriveau, R., and Ting, D. S.-K., "An experimental study on the interaction between free-stream turbulence and a wing-tip vortex in the near-field," Aerospace Science and Technology, Vol. 43, 2015, pp. 395-405. https://doi.org/10.1016/j.ast.2015.03.021

[10] Bailey, S. C. C., and Tavoularis, S., "Measurements of the velocity field of a wing-tip vortex, wandering in grid turbulence," Journal of Fluid Mechanics, Vol. 601, 2008, p. 281-315. https://doi.org/10.1017/S0022112008000694

[11] Bailey, S., H.K. Lee, B., and Tavoularis, S., "Effects of Free-Stream Turbulence on WingTip Vortex Formation and Near Field," Journal of Aircraft, Vol. 43, 2006, pp. 1282-1291. https://doi.org/10.2514/1.19433

[12] Stinebring, D. R., Farrell, K. J., and Billet, M. L., "The structure of a three dimensional vortex at high Reynolds number," Journal of Fluids Engineering, Vol. 113, 1983, pp. 496-503. https://doi.org/10.1115/1.2909524 
[13] Sarpkaya, T., "Trailing vortices in homogeneous and density-startified media," Journal of Fluid Mechanics, Vol. 136, 1983, pp. 85-109. https://doi.org/10.1017/S0022112083002074

[14] Shekarriz, A., Fu, T. C., Katz, J., Liu, H. L., and Huang, T. T., "Study of junction and tip-vortices using particle displacement velocimetry," AIAA Journal, Vol. 31, 1983, pp. 112-118. https://doi.org/10.2514/3.10894

[15] Anderson, E. A., and Lawton, T. A., "Correlation Between Vortex Strength and Axial Velocity in a Trailing Vortex," Journal of Aircraft, Vol. 40, No. 4, 2003, pp. 699-704. https://doi.org/10.2514/2.3148 URL https://doi.org/10.2514/2.3148.

[16] Nedić, J., Supponen, O., Ganapathisubramani, B., and Vassilicos, J. C., "Geometrical influence on vortex shedding in turbulent axisymmetric wakes," Physics of Fluids, Vol. 27, No. 3, 2015. https://doi.org/10.1063/1.4913573

[17] Chong, T. P., Vathylakis, A., Joseph, P. F., and Gruber, M., "Self-Noise Produced by an Airfoil with Nonflat Plate Trailing-Edge Serrations," AIAA Journal, Vol. 51, No. 11, 2013, pp. 2665-2677. https://doi.org/10.2514/1.J052344. URL https://doi.org/10.2514/1.J052344

[18] Nedić, J., and Vassilicos, J. C., "Vortex shedding and aerodynamic performance of airfoil with multiscale trailing-edge modifications," AIAA Journal, Vol. 52, No. 11, 2015, pp. 3240-3250. https://doi.org/10.2514/1.J053834.

[19] Prigent, S. L., Buxton, O. R. H., and Bruce, P. J. K., “Coherent structures shed by multiscale cut-in trailing edge serrations on lifting wings," Physics of Fluids, Vol. 29, No. 7, 2017, p. 075107. https://doi.org/10.1063/1.4995467, URL https: //doi.org/10.1063/1.4995467

[20] Thomareis, N., and Papadakis, G., "Effect of trailing edge shape on the separated flow characteristics around an airfoil at low Reynolds number: A numerical study," Physics of Fluids, Vol. 29, No. 1, 2017, p. 014101. https://doi.org/10.1063/1.4973811. URL https://doi.org/10.1063/1.4973811

[21] Tucker, V. A., "Drag reduction by wing tip slots in a gliding Harris' hawk, Parabuteo unicinctus," Journal of Experimental Biology, Vol. 198, No. 3, 1995, pp. 775-781. URL https://jeb.biologists.org/content/198/3/775

[22] Klein Heerenbrink, M., Warfvinge, K., and Hedenström, A., "Wake analysis of aerodynamic components for the glide envelope of a jackdaw (Corvus monedula)," Journal of Experimental Biology, Vol. 219, 2016, pp. 1572-1581. https: //doi.org/10.1242/jeb.132480

[23] KleinHeerenbrink, M., Johansson, L. C., and Hedenström, A., "Multi-cored vortices support function of slotted wing tips of birds in gliding and flapping flight," Journal of The Royal Society Interface, Vol. 14, No. 130, 2017, p. 20170099. https://doi.org/10.1098/rsif.2017.0099

[24] Klaassen van Oorschot, B., Tang, H. K., and W Tobalske, B., "Phylogenetics and ecomorphology of emarginate primary feathers," Journal of morphology, Vol. 278, 2017. https://doi.org/10.1002/jmor.20686

[25] Withers, P., “An aerodynamic analysis of bird wings as fixed aerofoils,” J. Exp. Biol., Vol. 90, 1981. 
[26] Hummel, D., "The aerodynamic characteristics of slotted wing-tips in soaring birds," Proceedings of the 17th International Ornithology Congress, CP849, Vol. 1, 1980, pp. 391-396.

[27] Pope, A., Low-Speed Wind Tunnel Testing, The McGraw Hill Companies, 1966.

[28] Benedict, L. H., and Gould, R. D., “Towards better uncertainty estimates for turbulence statistics," Experiments in Fluids, Vol. 22, No. 2, 1996, pp. 129-136. https://doi.org/10.1007/s003480050030, URL https://doi.org/10.1007/s003480050030

[29] Ramaprian, B. R., and Zheng, Y., "Measurements in Rollup Region of the Tip Vortex from a Rectangular Wing," AIAA Journal, Vol. 35, No. 12, 1997, pp. 1837-1843. https://doi.org/10.2514/2.59 URL https://doi.org/10.2514/2.59.

[30] Devenport, W. J., Rife, M. C., Liapis, S. I., and Follin, G. J., “The structure and development of a wing-tip vortex,” Journal of Fluid Mechanics, Vol. 312, 1996, pp. 67-106. https://doi.org/10.1017/S0022112096001929

[31] Engel, M. A., “A wind tunnel investigation of a wing-tip trailing vortex,” Masters thesis, 1995.

[32] Chow, J., Zilliac, G., and Bradshaw, P., "Initial Roll-Up of a Wingtip Vortex in: Proceedings of the aircraft wake vortices conference," Federal Aviation Administration, Vol. 2, 1992.

[33] Phillips, W. R., “The turbulent trailing vortex during roll-up,” Journal of Fluid Mechanics, Vol. 105, 1981, pp. $451-467$. https://doi.org/10.1017/S0022112081003285

[34] Ghimire, H. C., and Bailey, S. C. C., "An experimental investigation of wing-tip vortex decay in turbulence," Physics of Fluids, Vol. 29, No. 3, 2017, p. 037108. https://doi.org/10.1063/1.4979133 URL https://doi.org/10.1063/1.4979133

[35] Giuni, M., and B. Green, R., "Vortex formation on squared and rounded tip," Aerospace Science and Technology, Vol. 29, 2013, pp. 191-199. https://doi.org/10.1016/j.ast.2013.03.004.

[36] Katz, J., and Bueno Galdo, J., "Effect of roughness on rollup of tip vortices on a rectangular hydrofoil," Journal of Aircraft, Vol. 26, 1989, pp. 247-253. https://doi.org/10.2514/3.45753

[37] Green, S. I., and Acosta, A. J., “Unsteady flow in trailing vortices,” Journal of Fluid Mechanics, Vol. 227, 1991 , p. $107-134$. https://doi.org/10.1017/S0022112091000058

[38] Shekarriz, A., Fu, T. C., Katz, J., and Huang, T. T., "Near-field behavior of a tip vortex,” AIAA Journal, Vol. 31, No. 1, 1993 , pp. 112-118. https://doi.org/10.2514/3.11326 URL https://doi.org/10.2514/3.11326

[39] Baker, G. R., Barker, S. J., Bofah, K. K., and Saffman, P. G., "Laser anemometer measurements of trailing vortices in water," Journal of Fluid Mechanics, Vol. 65, No. 2, 1974, p. 325-336. https://doi.org/10.1017/S0022112074001418

[40] Jacquin, L., Fabre, D., Geffroy, P., and Coustols, E., "The properties of a transport aircraft wake in the extended near-wake field: an experimental study," 2001. https://doi.org/10.2514/6.2001-1038

[41] Jammy, S., Hills, N., and Birch, M., David, "Boundary conditions and vortex wandering," Journal of Fluid Mechanics, Vol. 747, 2014, p. 350-368. https://doi.org/10.1017/jfm.2014.169 
[42] Iungo, G., Skinner, P., and Buresti, G., "Correction of wandering smoothing effects on static measurements of a wing-tip vortex," Experiments in Fluids, Vol. 46, 2009, pp. 435-452. https://doi.org/10.1007/s00348-008-0569-2

[43] Batchelor, G. K., "Axial flow in trailing line vortices,” Journal of Fluid Mechanics, Vol. 20, No. 4, 1964 , p. 645-658. https://doi.org/10.1017/S0022112064001446 